\title{
Single Cell Profiling of Acute Kidney Injury Reveals Novel Transcriptional Signatures, Mixed Identities and Epithelial-to-Stromal Crosstalk
}

Valeria Rudman-Melnick ${ }^{1}$, Mike Adam ${ }^{1}$, Andrew Potter ${ }^{1}$, Saagar M. Chokshi², Qing Ma², Keri A. Drake $^{3}$, Meredith P. Schuh ${ }^{2}$, J. Matthew Kofron ${ }^{1}$, Prasad Devarajan ${ }^{2}$ and S. Steven Potter ${ }^{1,4, *}$

1'Division of Developmental Biology, Cincinnati Children's Medical Center, Cincinnati, OH 45229, USA 2Division of Nephrology and Hypertension, Cincinnati Children's Medical Center, Cincinnati, $\mathrm{OH}$ 45229, USA

${ }^{3}$ Division of Pediatric Nephrology, UT Southwestern Medical Center

${ }^{4}$ Lead Contact

*Correspondence: Steve.Potter@cchmc.org 


\section{Summary}

Acute kidney injury (AKI) is a rapid decline of renal function, with an incidence of up to $67 \%$ of intensive care unit patients. Current treatments are merely supportive, emphasizing the need for deeper understanding that could lead to improved therapies. We used single cell RNA sequencing, in situ hybridization and protein expression analyses to create comprehensive renal cell specific transcriptional profiles of multiple AKI stages. We revealed that AKI induces marked dedifferentiation, renal developmental gene activation and mixed identities in injured renal tubules. Moreover, we identified potential pathologic crosstalk between epithelial and stromal cells, and several novel genes involved in AKI. We also demonstrated the definitive effects of age on AKI outcome, and showed that renal developmental genes hold a potential as novel AKI markers. Moreover, our study provides the resource power which will aid in unraveling the molecular genetics of AKI.

\section{Keywords}

acute kidney injury, single-cell, Sox4, Cd24a, lineage infidelity, cellular interactions 


\section{Introduction}

Acute kidney injury (AKI) is a group of syndromes characterized by abrupt renal function decline associated with pronounced mortality and co-morbidities in other organs (Hoste et al., 2018). AKI is a common condition with estimated prevalence ranging from $<1 \%$ to $66 \%$ and increasing trends of hospitalization number (Pavkov et al., 2018), which represents a global burden to the healthcare system. Moreover, AKI episodes can initiate or exacerbate chronic kidney disease (CKD), a debilitating condition requiring lifelong dialysis or renal transplantation (Hsu and Hsu, 2016). Thus, examining the molecular signaling pathways involved in early AKI response is paramount for treatment development.

Single cell RNA sequencing (scRNA-seq) has been used to thoroughly characterize the gene expression profiles of normal cells types of the developing and adult kidney for both mouse and human (Brunskill et al., 2014; Combes et al., 2019; Hochane et al., 2019; Ransick et al., 2019; Wang et al., 2018). In this study, we present the first comprehensive atlas of renal expression changes at multiple stages of AKI progression at the single cell level. Since disrupted renal perfusion represents one of the most clinically relevant causes of kidney disease (Yang et al., 2010), we used the unilateral ischemia/reperfusion (UIR) model of AKI which allows for inducing severe renal injury without significant mortality (Le Clef et al., 2016). We identified unique injury response gene expression signatures of all renal cell populations. Proximal tubule cells showed a rapid reduced expression of differentiation markers such as S/c34a1, while showing increased expression of genes normally expressed during development, such as Sox4 and Cd24a. Particularly striking, many of the injured cells showed simultaneous expression of markers of multiple nephron cell types, consistent with 
reversion to an early developmental stage (Brunskill et al., 2014; Magella et al., 2018). We identified several novel genes which were not previously reported to play a role in AKI response. Further, we found that injured tubules expressed genes normally associated with the stromal lineage. Analysis of ligand/receptor interactions revealed potential pathologic crosstalk between epithelial and stromal cells. We also examined the AKI response as a function of age of injury, finding that young mice show a much more rapid recovery with greatly reduced long term fibrosis. The results show that renal developmental genes represent potential novel AKI markers.

\section{Results and Discussion}

\section{Single Cell RNA Sequencing Reveals Proximal Tubule Dedifferentiation and Mixed Identity}

\section{Cells in the Ischemia Reperfusion induced AKI}

To examine the molecular and cellular nature of acute kidney injury (AKI) response, we used the clinically relevant model of Unilateral Ischemia/Reperfusion (UIR) AKI (Le Clef et al., 2016) (Figure 1A). To evaluate the transcriptional changes in renal cell populations over the course of AKI, we performed single cell RNA-seq (scRNA-seq) on renal cell suspensions from Swiss-Webster 4 weeks old male mice at Days 1, 2, 4, 7, 11 and 14 after UIR (Figure 1B). We carried out scRNA-seq using Drop-Seq as previously described (Adam et al., 2017; Macosko et al., 2015; Magella et al., 2018), and used unsupervised clustering (Stuart et al., 2019) to generate Uniform Manifold Approximation and Projection (UMAP) plots which separated the cells into distinct groups (Fig. 1C). We identified resulting cell clusters including Nphs2-positive podocytes, proximal tubule (SIc34a1), distal tubule (Slc12a3), loop of Henle (Slc12a1), collecting duct (Aqp2 for principal and Foxi1 for intercalated cells) 
A

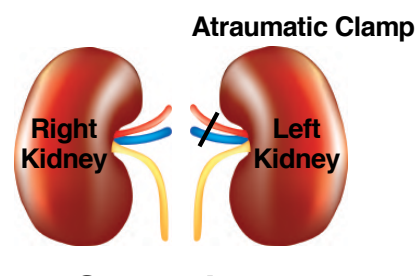

C
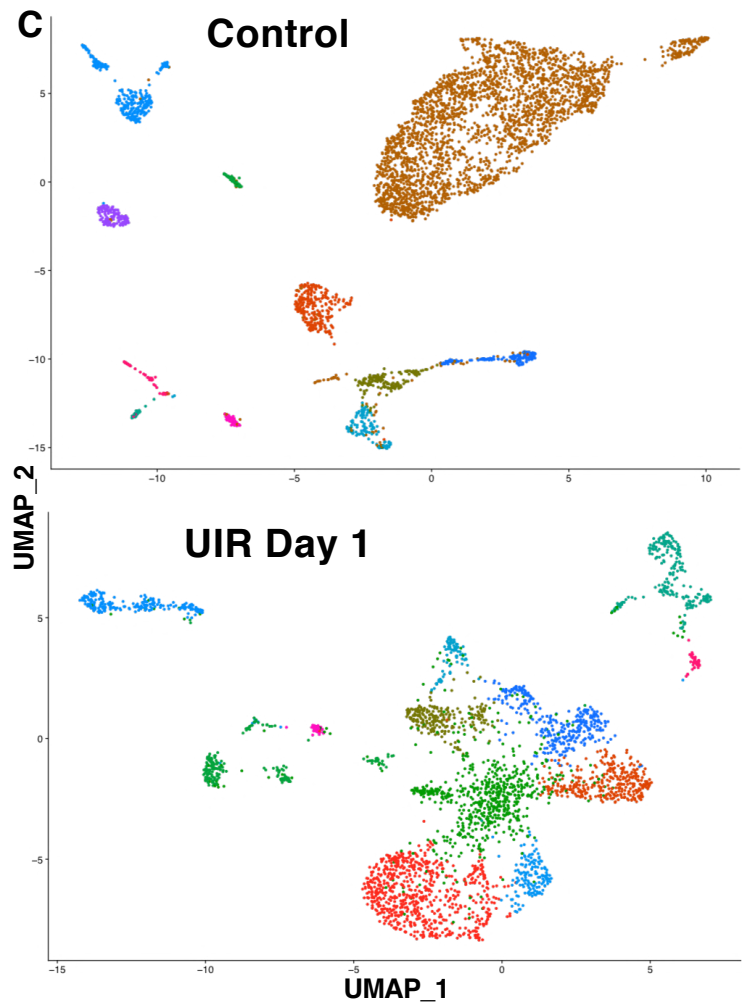

F
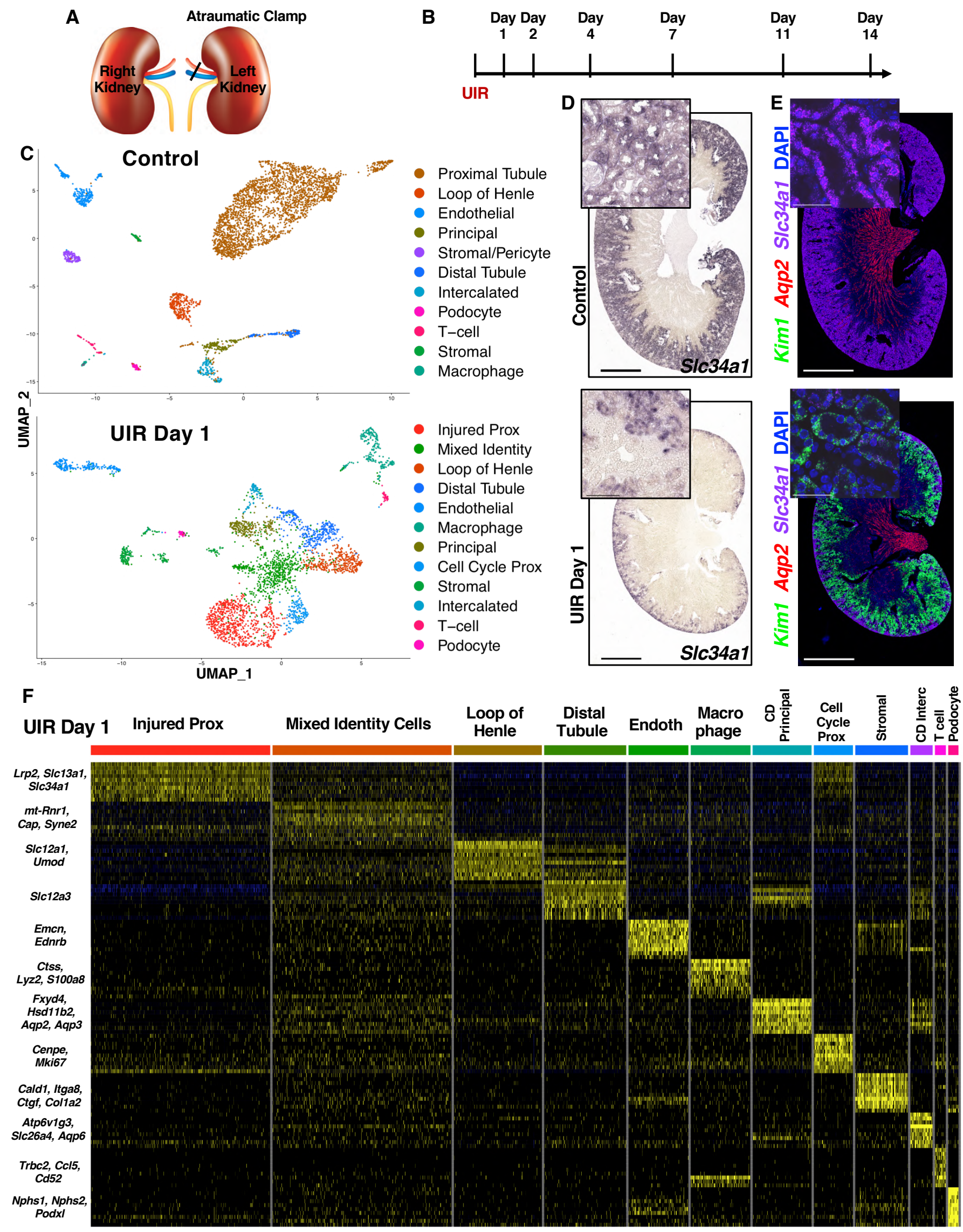

Injured Prox

- Mixed Identity

- Loop of Henle

- Distal Tubule

- Endothelial

- Macrophage

- Principal

- Cell Cycle Prox

- Stromal

Intercalated

- T-cell

- Podocyte
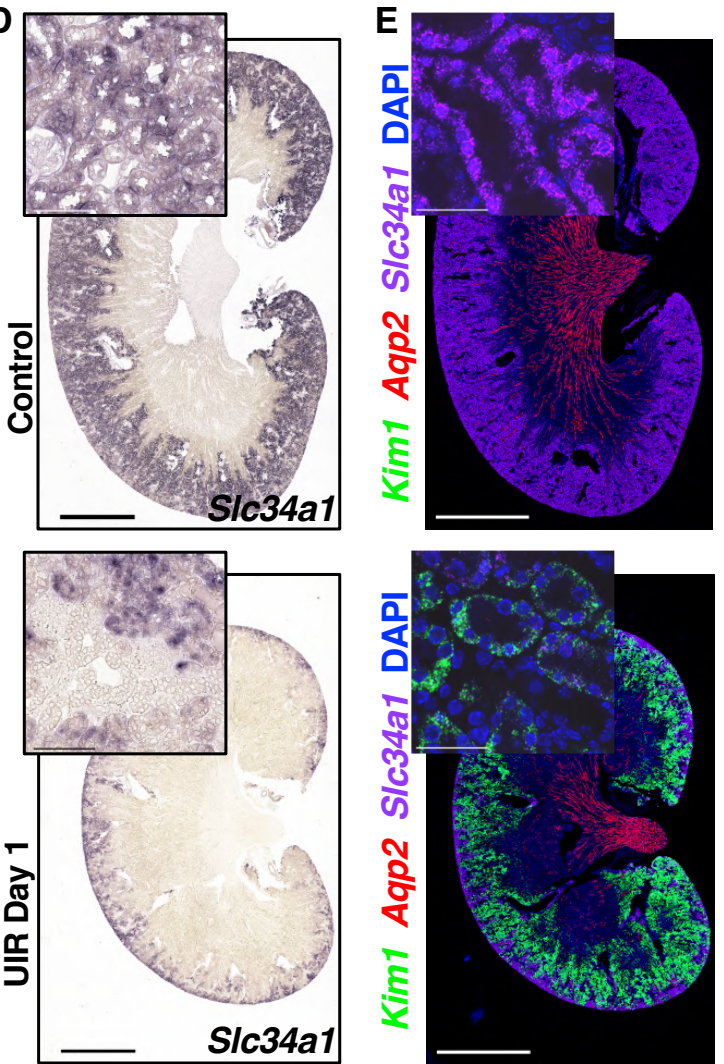


\section{Figure 1. Single Cell RNA Sequencing Reveals Proximal Tubule Dedifferentiation and Mixed}

\section{Cell Identity in the Ischemia Reperfusion induced AKI}

(A) UIR procedure scheme.

(B) The experimental timeline.

(C) UMAP plots show the renal cell populations in the Control and UIR Day 1.

(D) SIc34a1 CISH, Control and UIR Day 1, 4x, 40x.

(E) Kim1 (green), Aqp2 (red), Slc34a1 (purple) RNAscope, Control and UIR Day 1, 4x, 60x.

(F) Heatmap shows the relative marker gene expression in UIR Day 1 of renal cell types. Endoth, Endothelial; CD, Collecting Duct; Interc, Intercalated. The Mixed Identity Cells show stochastic expression of markers of many different cell types.

Scale: $4 \mathrm{x}-2500 \mu \mathrm{m}, 40 \mathrm{x}-100 \mu \mathrm{m}, 60 \mathrm{x}-25 \mu \mathrm{m}$.

Related to Figures S1, S2, Table S1.

as well as endothelial cells $(P e c a m 1)$, pericytes $(P d g f r \beta)$, macrophages $(C d 68)$, T cells $(C d 3 g)$, and stromal/pericytes cells (Pdgfrb) (Figure 1C and S1A).

We first compared gene expression patterns of control and UIR Day 1 kidneys for each cell type (Table S1). In the normal kidney, the renal sodium-dependent phosphate transporter Slc34a1 marks mature proximal tubules, which represent the predominant cell type in the renal cortex, as shown by both scRNA-seq and chromogenic in situ hybridization (CISH) (Figures 1C and 1D, S1A). UIR, however, resulted in a markedly reduced Slc34a1-positive mature proximal tubule population (Figures $1 \mathrm{C}$ and $\mathrm{S} 1 \mathrm{~B})$. We validated this finding with $\mathrm{CISH}$ performed on an independent cohort of 4 weeks old Swiss-Webster mice treated with the identical UIR. While the control mice abundantly expressed Slc34a1 in the cortex and inner medulla, the UIR Day 1 exhibited significantly reduced SIc34a1 levels, with remaining expression only in the outer cortex (Figure 1D). This observation is consistent with the previously reported injury-induced proximal tubule dedifferentiation (Kramann et al., 2015; Kusaba et al., 2014). Moreover, we identified the abundant elevation of kidney injury molecule-1 (Kim1, a.k.a. Havcr1) in the cortex and outer medulla of the UIR Day 1 kidney compared to control, using fluorescent in situ hybridization (FISH, a.k.a. RNAscope) (Figure 1E). Kim1 is a clinically 
recognized AKI marker (Khreba et al., 2019); as we observed and others (Bonventre, 2008; Gauer et al., 2016) have shown, Kim1 is predominantly expressed in the UIR Day 1 injured proximal tubules and is nearly absent in the Control (Figure S1A). Based on Kim1 and Slc34a1 distribution, we identified the cluster of "Injured Prox", defined by the most prominent Kim1 and lowered Slc34a1 levels (Figure 1C, S1B). The cluster of "Cell Cycle Prox" was identified based on the high expression of cell cycle markers, including the proliferation marker Mki67 (Figure S1B). Next, we identified the loop of Henle, distal tubule, collecting duct and found that they all are highly enriched with Lipocalin 2 (Lcn2), another clinically used AKI marker specific for the distal segments of the nephron tubule (Schmidt-Ott et al., 2007; Singer et al., 2016; Sun et al., 2018) (Figures S1B and S1C). Histological comparison of UIR Day 1 vs Control using Hematoxylin \& Eosin (H\&E) staining revealed signs of pronounced tubular epithelial injury, including renal tubular dilation, epithelial flattening and cast formation (Figure S1D). Thus, we showed that UIR Day 1 comprises all classical AKI features, including marked mature cell dedifferentiation and injury in multiple nephron tubule segments (Basile et al., 2012; Kudose et al., 2018).

To identify the molecular signaling pathways and biological processes which might play a role in the early $\mathrm{AKI}$ response, we performed functional enrichment gene analysis and candidate gene prioritization using the ToppGene Suite (Chen et al., 2009). Gene Ontology (GO) analysis of UIR Day 1 injured proximal tubules showed substantial reduction of proximal tubule metabolism, including oxidation-reduction processes (SIC37a4, SIc25a2, Aco1, Aco2, Cyp2e1, Miox) and fatty acid catabolism (Acadl, Acadm, Cd36, Cpt1a, Crot, Slc27a2), which are the key proximal tubule metabolic pathways (Kang et al., 2015; Simon and Hertig, 2015) (Figure S1E, Table S1). We also observed the UIR induced reduction of transmembrane transport pathways (Slc34a1, Aqp1, Lrp2, Slc7a13, Slc22a6) crucial for normal proximal tubule homeostatic functions. Conversely, UIR caused elevation of apoptotic processes (Acin1, Tnfrsf12a, Clu, Nfkbia, Lgals1, Ctsd), cell cycle regulation (Ccnd1, 
Ccn/1, Ctnnb1, Pcna), granulocyte activation and cytokine response (C3, S100a11, Lcn2, Cstb, Anxa2), and cellular response to oxygen (Mgst1, Gpx3, Aoc1) in the "Injured Prox" (Figure S1E, Table S1).

Notably, we found that AKI not only induces dedifferentiation, but causes an ambiguous transcriptional identity and ectopic expression of mature defined renal cell populations markers. In the UIR Day 1, we noticed a large population of cells with overlapping expression of the collecting duct marker Aquaporin 2 (Aqp2) (Yu et al., 2014) and the loop of Henle marker Uromodulin (Umod) (Tokonami et al., 2018) (Figure S2A). These "Mixed Identity Cells" were located between proximal tubules, loop of Henle, distal tubule and collecting duct on the UMAP (Figures 1C, S2A), suggesting the intermediate character. Also, the scRNA-seq data showed that many of these cells expressed both Kim1 and Lcn2 (Figure S2B) which mark proximal and distal nephron tubule segment injury, respectively. A heat map revealed the apparent mixed identity, showing that these cells exhibit stochastic expression of markers of many different kidney cell types (Figure 1F), including principal (Fxyd4, Hsd11b2, Aqp2, Aqp3) and intercalated (Atp6v1g3, Atp6v0d2, Slc26a4, Aqp6) cells of the collecting duct (Chen et al., 2017), proximal tubule (Slc34a1), loop of Henle (Slc12a1, Umod) and distal tubule (Slc12a3), which was not observed in the control (Figure S2C). This scRNA-seq observation was reproduced in an additional independent cohort of validation animals. To detect the mixed identity cells, we hybridized Slc34a1, Slc12a1, Slc12a3 and Aqp2 RNAscope probes to the UIR Day 1 and Control kidney sections and found that UIR result in the remarkable presence of cells expressing multiple epithelial compartments markers. Prominently, IMARIS quantification revealed significantly increased numbers of Aqp2, Slc12a3 and Slc12a1 transcripts in the tubules exhibiting lowered Slc34a1 levels (Figures 2A-2D), as predicted by scRNA-seq. The presence of Aqp2 transcripts in Slc34a1-positive tubules demonstrates the marked plasticity of adult renal injured cells, since the proximal tubule (S/c34a1) and the collecting duct (Aqp2) arise from the different 
developmental lineages, the metanephric mesenchyme and the nephric duct respectively (Costantini and Kopan, 2010; McMahon, 2016). Of interest, we also noticed that AKI not only induces the presence of the mixed identity cluster, but also results in inappropriate ectopic gene expression in cells that clustered as a defined cell type. For example, the RNAscope showed elevated Aqp2 transcripts in the Kim1-positive injured proximal tubules (Figure 2E).

Noteworthy, the AKI also resulted in ambiguous epithelial/stromal identities. The heat map shows that the "Mixed Identity Cells" exhibit increased expression of stromal genes, particularly activated fibroblast markers (Ctgf, Col1a2, Cald1) (Shieh et al., 2019; Wu et al., 2018; Zhang et al., 2017b) (Figure 1F) and genes related to the cell shape change and extracellular matrix remodeling (Sparc, Lgals3, Spp1, Myh9, S100a10) (Table S1), giving evidence for a more mesenchymal phenotype acquired by these cells in response to the injury. Epithelial-to-mesenchymal plasticity is a crucial contributor to the kidney injury and fibrosis (Humphreys, 2018; Lovisa et al., 2016). In contrast, this cluster exhibited much weaker expression of genes associated with more distantly related cell types, like macrophages and T-cells (Figure 1F). Overall, our data reveals that AKI induces apparent cellular plasticity between adult renal cell types. We previously showed that lineage infidelity, or mixed cell identity, occurs in the developing kidney, with the unexpected stochastic expression of markers of multiple differentiated renal cell types in single cells of the E11.5 MM, cap mesenchyme and later renal vesicles (Brunskill et al., 2014; Magella et al., 2018). However, this cellular plasticity between renal epithelial compartments, including those of different developmental origins, to our knowledge had not been previously observed in the adult AKI. 

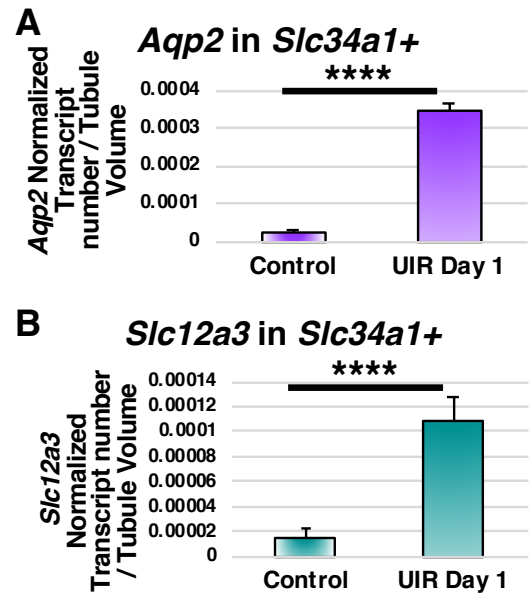

C Slc12a1 in Slc34a1+

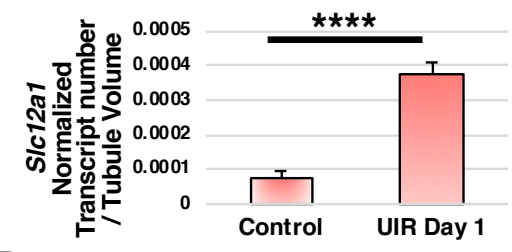

D
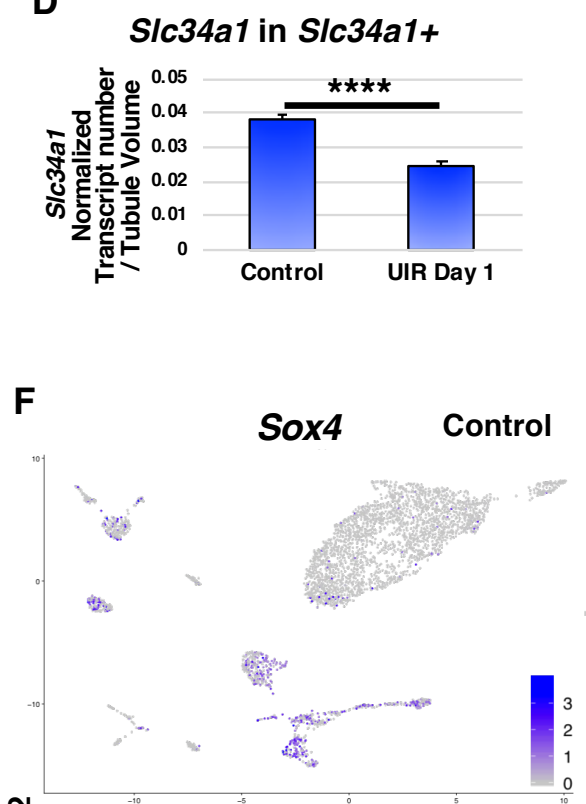

$\frac{N}{5}$

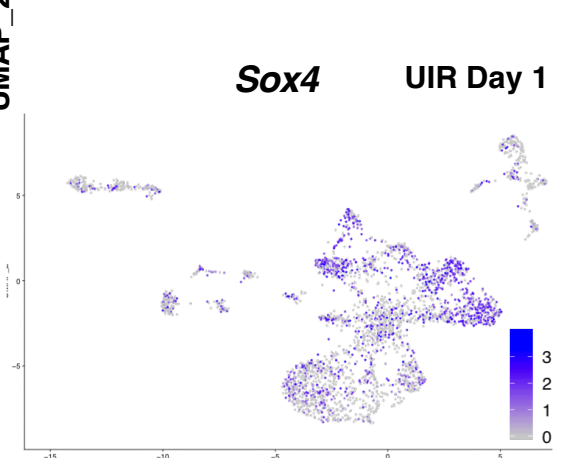

UMAP_1

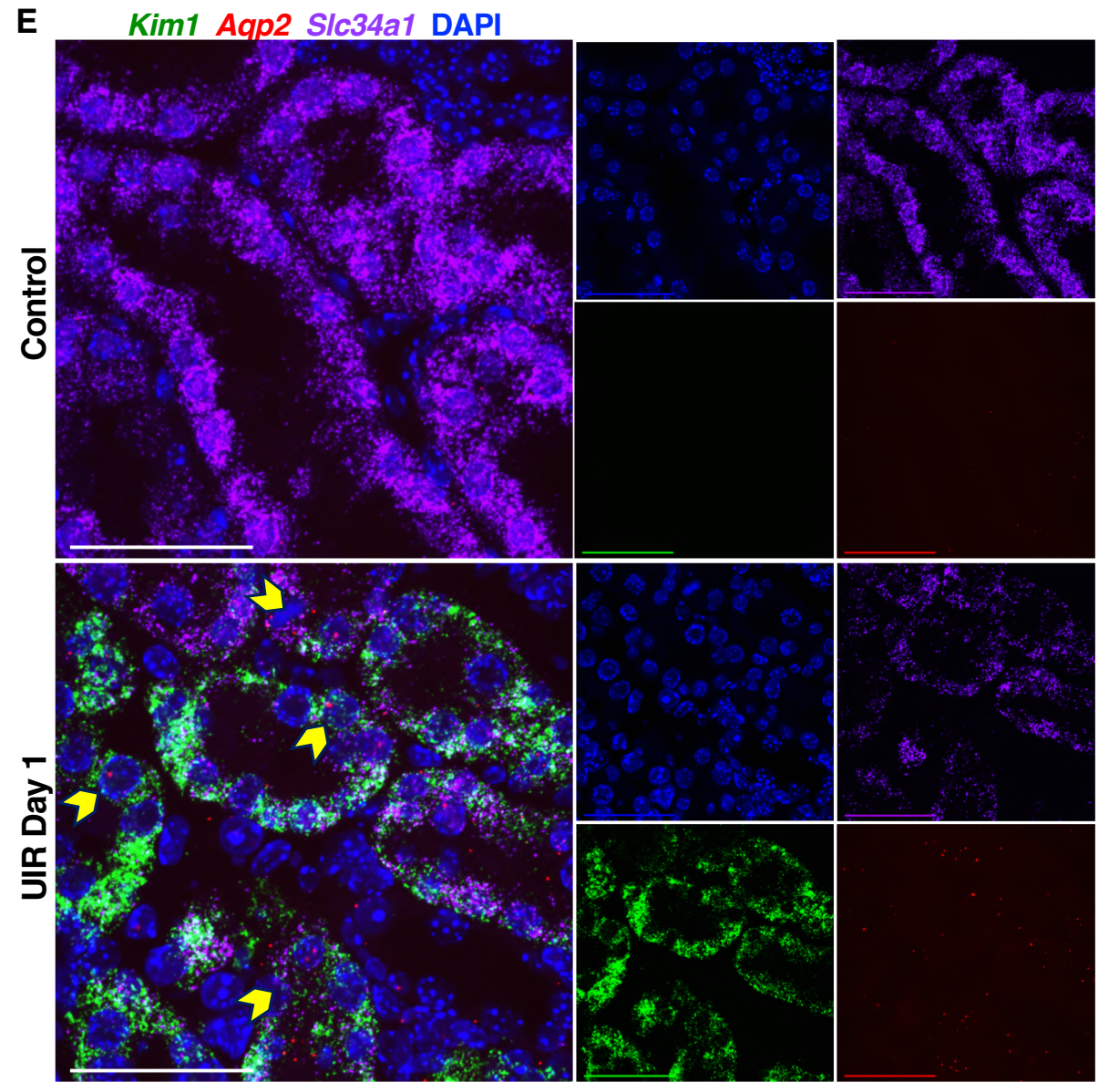

H Cd24a

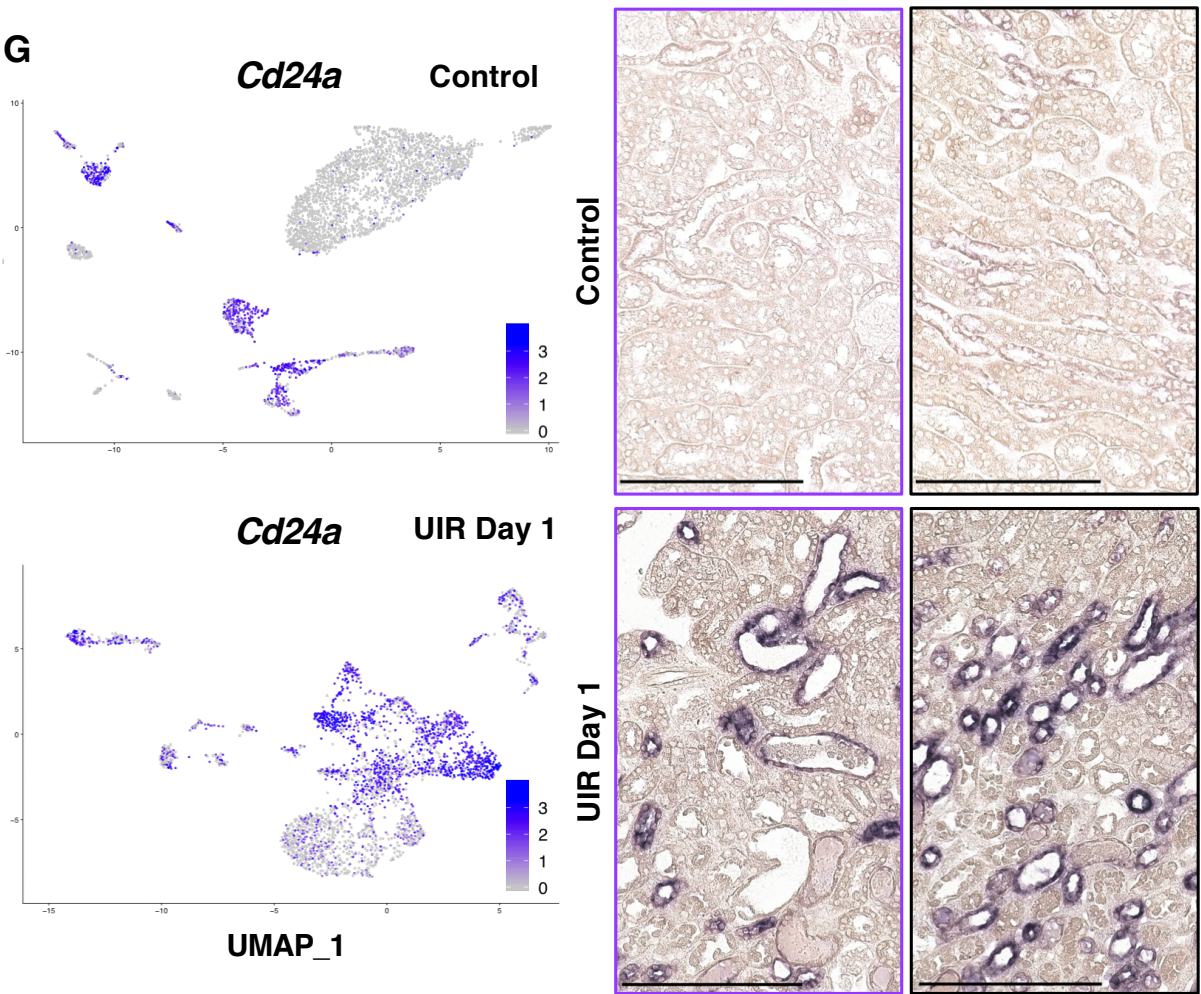




\section{Figure 2. AKI Induces Renal Developmental Program Re-Activation in the Adult Kidney}

(A-D) IMARIS quantification of Aqp2, Slc12a3, SIc12a1 and Slc34a1 in the UIR Day 1 vs Control renal tubules, $\mathrm{n}=12 \mathrm{Z}$ stacks (50-70 tubules) per group, Mean \pm SEM, Student's $t$ test, ${ }^{\star \star \star \star} \mathrm{p}<0.0001$.

(E) Representative images of Kim1 (green), Aqp2 (red), Slc34a1 (purple) RNAscope, Aqp2 in Slc34a1- or Kim1-positive UIR Day 1 proximal tubules (yellow pointers). Maximal Intensity Projection from Z-stacks, 60x 1.5 Nyquist zoom, scale $50 \mu \mathrm{m}$.

( $F$ and $G$ ) UMAPs show the elevated Cd24a and Sox4 expression in UIR Day 1. Cell cluster identities are as defined in Figure 1.

(H) $\mathrm{CISH}$ shows the elevated Cd24a expression in the UIR Day 1 renal tubules; 40x, cortex, purple frames (left); medulla, black frames (right); scale $100 \mu \mathrm{m}$.

Related to Figure S2, Table S1.

\section{AKI induces Renal Developmental Program Re-Activation in the Adult Kidney}

Next, we further analyzed the gene expression signatures of injured renal cells. The GO analysis of the "Mixed Identity Cells" revealed the enrichment of renal developmental pathways (Figure S2C, Table S1). Notably, we identified the Transcription Factor SRY-related HMG Box-4 (Sox4) (Figure 2F), implicated in the urogenital system, kidney and epithelium development by GO analysis.

Previous work showed that Sox4 is strongly expressed in nephron progenitors and ureteric bud during early and late renal development as well as in the newborn kidney (Huang et al., 2013; Yu et al., 2012). Moreover, Six2Cre;Sox4 mutants exhibit reduced nephron number and proteinuria postnatally, which progresses to ESRD defined by glomerulosclerosis and proximal tubular injury (Huang et al., 2013), showing that Sox4 is essential for proper nephrogenesis. However, Sox4 has no known role in AKI. We also observed that that the "Mixed Identity Cells" were enriched with Signal Transducer Cd24 (Cd24a) (Figure 2G, Table S1), which encodes a cell-surface sialoglycoprotein expressed in the uninduced metanephric mesenchyme (MM) of the developing kidney (Challen et al., 2004). Human Cd24 is also implicated in kidney development and tubular epithelial differentiation (Ivanova et al., 2010; Lazzeri et al., 2007). We observed that Cd24a is also elevated in the adult injured proximal tubules (Figure 2G), consistent with previous reports (Kramann et al., 2015; Kusaba 
et al., 2014). Moreover, the UMAPs showed that both Sox4 and Cd24a were also enriched in the loop of Henle and distal tubule, and in the principal and intercalated cells of the collecting duct (Figures $2 \mathrm{~F}$ and $2 \mathrm{G}$ ). With $\mathrm{CISH}$, we validated the elevation of $\mathrm{Cd} 24 \mathrm{a}$ in both cortical and medullary renal tubules (Figure 2H). Noticeably, the AKI induced Sox4 and Cd24a elevation recapitulated their developing kidney spatial expression patterns (Harding et al., 2011; McMahon et al., 2008; Yu et al., 2012), which demonstrates the profound nature of renal developmental program re-activation and mature renal cell dedifferentiation caused by the injury.

Next, we examined cell populations and molecular changes which occur later after AKI induction. Analysis of cell clusters from UIR Day 2 revealed persistent kidney injury, indicated by continued elevated Kim1 expression and the decreased number of strongly positive Slc34a1 proximal tubules (Figures 3A-3C). scRNA-seq also revealed the Mixed Identity cluster, characterized by marked gene expression heterogeneity and renal developmental gene elevation, along with the clusters of macrophages and T-cells, showing the enduring inflammation (Figure 3A). Histological analysis showed exacerbated renal tubular damage, compared to day 1 , exemplified by pronounced epithelial flattening, tubular casts and dilation (Figure S3A). Renal tubular injury persisted through UIR Day 4 and 7; however, we observed signs of recovery, including increased Slc34a1 expression and reduced total kidney Kim1 and Lcn2 RNA and protein levels (Figures 3A-3C, S3A-S3C). Consistent with previous work showing the role of macrophages in kidney injury resolution (Lee et al., 2011), we observed that both timepoints exhibited large macrophage infiltration (Figure 3A). The mixed identity cells were no longer present at Day 7; however, we identified the "Dedifferentiated Prox" cluster (Figure 3A, Table S2), which expressed the proximal tubular genes Aqp1, a water-transporting protein (Hara-Chikuma and Verkman, 2006), Slc7a12, a cationic amino acid transporter (Rinn et al., 2004), and Cdh2 (N-Cadherin) (Nurnberger et al., 2010; Prozialeck et al., 2004). However, this 
A
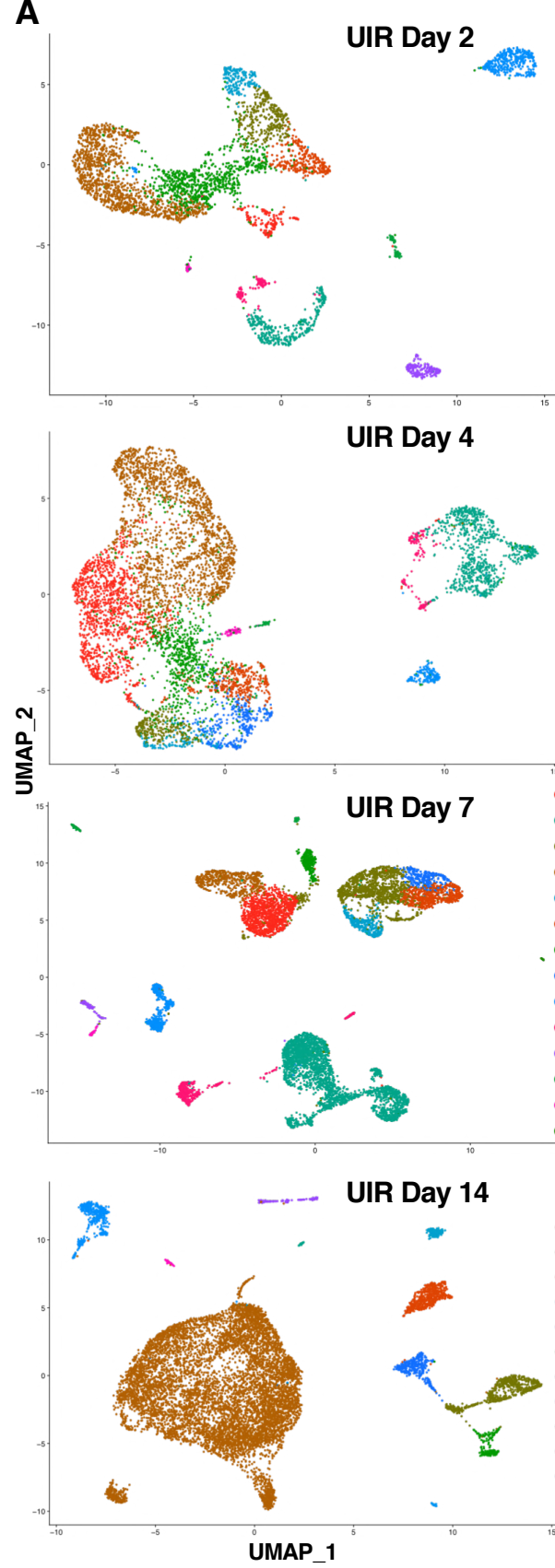

D

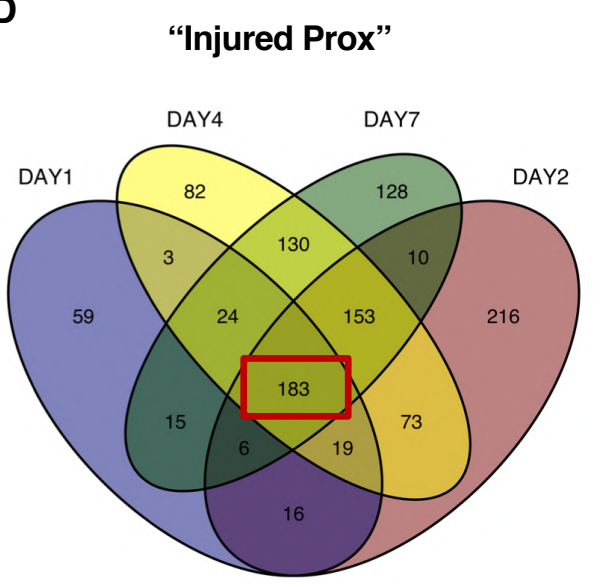

\section{B}

- Mixed Identity

- Endothelial

- Macrophage

- Principal

- Loop of Henle / Distal Tubule

- Intercalated

- Injured Prox

- T-cell

- Stromal

- Podocyte

- Injured Prox

- Mixed Identity

- Proximal Tubule

- Macrophage

Loop of Henle

- Distal Tubule

- Principal

- Endothelial

- Intercalated

- T-cell

- Podocytes

- Stromal

- Injured Prox

- Macrophage

- Principal

- Proximal Tubule

- Intercalated

- Loop of Henle

- Dedifferentiated Pro

- Distal Tubule

- Endothelial

- T-cell

- Pericyte/Stromal

- Stromal

- Podocyte

- Ureter

- Proximal Tubule

- Principal

- Loop of Henle

- Endothelial

- Distal Tubule

- Intercalating

- Pericyte/Stromal

- Macrophage/T-cell

- Podocyte

- Stromal

E

"Mixed Identity Cells"
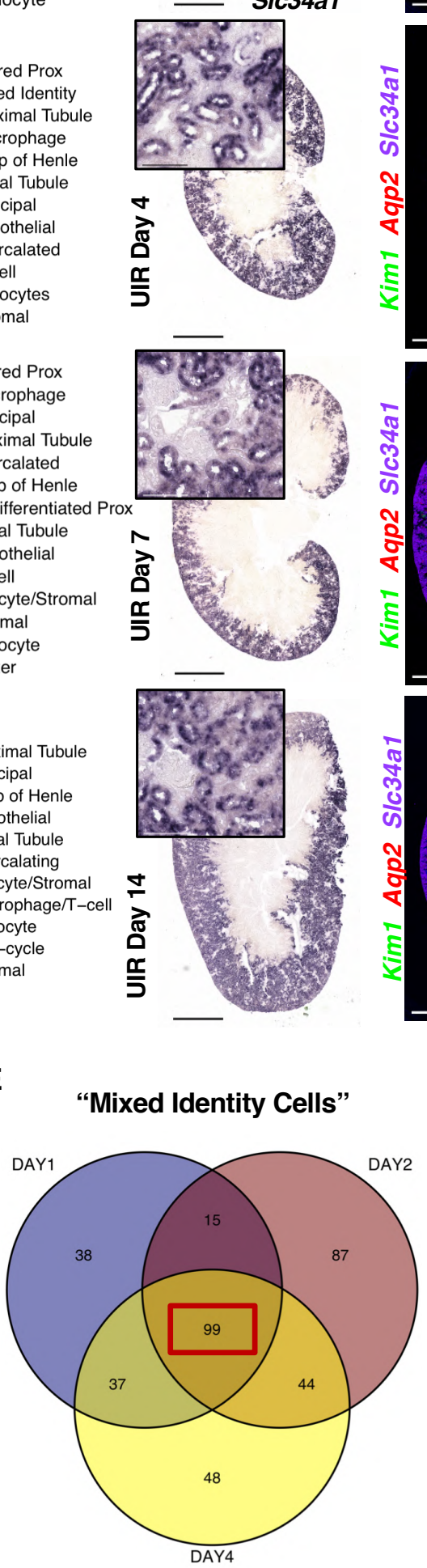
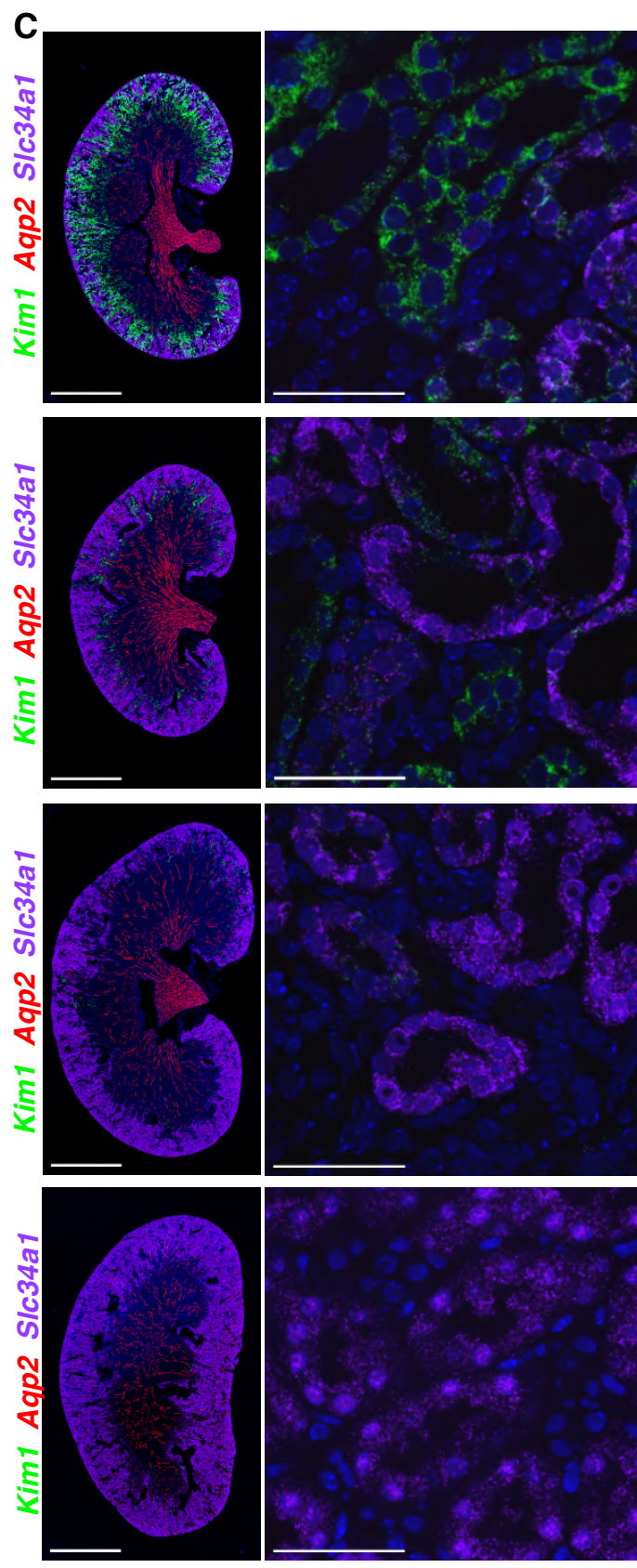

F

$\square$ Sox4 $\square C d 24 a$

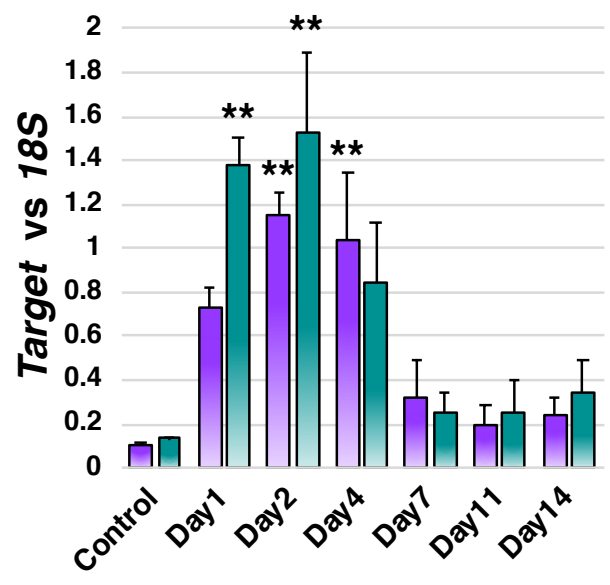




\section{Figure 3. scRNA-seq Reveals a Persistent Developmental Signature over the Course of AKI}

(A) UMAPs show renal cell populations in the UIR Day 2, 4, 7 and 14. Day 11 is not shown, as it was very similar to Day 14.

(B) SIc34a1 CISH, UIR Day 2, 4, 7 and 14, 4x, 40x.

(C) Kim1 (green), Aqp2 (red), Slc34a1 (purple) RNAscope, UIR Day 2, 4, 7 and 14. 4x, 60x.

(D) Venn diagram shows the genes elevated in the "Injured Prox" at UIR Day 1, 2, 4 and 7. Injured proximal tubule gene expression patterns were compared to control proximal tubule.

(E) Venn diagram shows the genes elevated in the "Mixed Identity Cells" at UIR Day 1, 2 and 4. Mixed identity cell gene expression patterns were compared to control proximal tubule.

(F) qPCR shows Sox4 and Cd24a expression over the AKI course, Mean \pm SEM, one way ANOVA with Bonferroni, ** pValue $<0.01$ compared to Control.

Scale: $4 \mathrm{x}-2500 \mu \mathrm{m}, 40 \mathrm{x}-100 \mu \mathrm{m}, 60 \mathrm{x}-25 \mu \mathrm{m}$.

Related to Figure S3, Table S2, S3.

cluster also showed a strong renal developmental signature, including the elevation of Sprouty1, Nephronectin, and the Forkhead-box transcription factor Foxc1, which all are crucial for the kidney induction (Basson et al., 2005; Kume et al., 2000; Linton et al., 2007). scRNA-seq also showed increased Notch2, a crucial regulator of nephron induction, segmentation and proximal tubule epithelium development (Chung et al., 2016; Sirin and Susztak, 2012), and Notch ligand Jag1, in the "Dedifferentiated Prox" (Table S2). Notably, recent work from the Susztak group shows the role of Notch2 in the collecting duct cell plasticity associated with metabolic acidosis (Park et al., 2018). This population also expressed Osr2, which is present in the early proximal tubule, but not in the adult cortical tubule (Harding et al., 2011; McMahon et al., 2008); and the metanephric mesenchyme marker Cd24a mentioned above. This developmental signature gives evidence for a dedifferentiated proximal tubule state.

Both scRNA-seq and RNAscope showed further kidney recovery at UIR Day 11 and 14, defined by restored SIc34a1 expression, absent Kim1-positive injured proximal tubules and decreased immune infiltration (Figures S3D-S3F and 3A-3C). Injury resolution was confirmed with H\&E staining (Figure 
S3A). The molecular analysis performed on all experimental time points showed the decrease of kidney injury markers Kim1 and Lcn2 over the course of AKI recovery, which returned back to normal by Day 14 (Figures S3B and S3C). While the small "Dedifferentiated Prox" cluster was still present at UIR Day 11, the kidney developmental signature was markedly weaker at this point (Table S2); these cells were not present at UIR Day 14. Similar to the Control kidney, UIR Day 14 proximal tubules were enriched with transmembrane transport and metabolic pathways, as the GO shows (Figure S3G), which reflects the normal proximal tubule homeostatic functions. These experiments examined multiple stages of AKI progression, thereby providing a powerful resource to dissect the gene expression changes in multiple cell populations involved in kidney injury and recovery.

Since proximal tubules are crucial contributors to the kidney injury response (Takaori et al., 2016), we further examined the gene expression changes characterizing the proximal tubular injury over the AKI course. We performed differential gene expression analysis of the "Injured Prox" compared to control at UIR Days 1, 2, 4 and 7, with a Venn diagram showing 183 common shared overlapping genes (Figure 3D). Noteworthy, the overlapping genes were enriched with pro-inflammatory pathways, such as leukocyte activation and degranulation (Lcn2, Lgals1, Anxa2, S100a11, Cstb) and viral process (Kim1, Ifi27, Cd74, Anxa2) (Table S3). Of particular interest, we identified the interferon-response gene Ifi27, encoding interferon alpha inducible protein 27 , previously associated with drug-induced nephrotoxicity (Dieterich et al., 2009), among the genes shared by the "Injured Prox" of UIR Day 1, 2, 4 and 7 (Table S3). Moreover, we noticed the persistent interferon-response gene signature (Ifit2, Ifit3, Ifitm3, Ifi44) not only in the injured proximal tubules, but also in the collecting ducts and distal tubules (Table S1). Interferon signaling is known to play a role in renal inflammatory and autoimmune pathologies (Anders et al., 2010), and, as we and others (Wu et al., 2019) report, the renal epithelium acquires a pro-inflammatory phenotype in response to the enduring injury. Remarkably, we identified that renal developmental genes $\mathrm{Cd} 24 \mathrm{a}$ and Sox4 marked the injured proximal tubules throughout the 
AKI course, as the differential gene expression analysis of the UIR Day 1, 2, 4 and 7 "Injured Prox" UIR Day 1, 2, 4 and 7 shows (Table S3).

Next, we examined the gene expression changes occurring in the "Mixed Identity Cluster" over the AKI course. The differential gene expression analysis of the genes elevated in the mixed identity cells compared to control proximal tubules at UIR Days 1,2 and 4 identified 99 genes common at all time points, as the Venn diagram shows (Figure 3E, Table S3). We identified Umod (loop of Henle) and Aqp2 (collecting duct) among the overlapping genes, consistent with the mixed identity. GO analysis revealed elevation of cytokine response (Ifi27, Ifitm3, Anxa2, Lcn2), vesicle fusion (Sparc, Tubb5, Tagln2, Lgals3) and programmed cell death regulation (Clu, Nfkbia, Ubb, Pea15a, Lgals1, Ctsd) (Figure S3H). Importantly, we noticed the significant elevation of Clusterin (Clu), in many injured kidney cell populations, including the "Mixed Identity Cells", proximal tubules and collecting duct (Table S1). Clu expression might play a protective role in the kidney injury, since Clu deficiency results in worsened $\mathrm{AKI}$ outcome and elevated fibrosis (Guo et al., 2016). Previous work (Dieterle et al., 2010) reported urinary clusterin as a kidney injury marker, along with cystatin $\mathrm{C}(\mathrm{Cst3})$ and $\beta 2-$ microglobulin (B2m), two other AKI induced genes the scRNA-seq identified (Tables S1, S3). Importantly, we found that kidney developmental genes marked the "Mixed Identity Cells" over the AKI course (Figure S3H). Particularly, ToppCluster analysis of the genes shared by the "Mixed Identity Cells" at UIR Day 1, 2 and 4 (Kaimal et al., 2010) identified Cd24a and Sox4 along with Aqp2 (collecting duct) and Umod (loop of Henle) mentioned above (Figure S3I). pPCR performed on the independent animal cohort validated the injury inducible Cd24a and Sox4 elevation shown by the scRNA-seq, which reversed by UIR Day 14, consistent with kidney recovery (Figure 3F). Overall, we demonstrated that renal developmental genes label renal epithelial injury, particularly injured proximal tubules and mixed identity cells, throughout the AKI course. 


\section{Sox4 and Cd24a Label the Proximal and Distal Tubule Injury}

Then, we further examined the renal developmental gene expression in the injured kidney. As scRNA-seq found and qPCR validated, Sox4 was elevated in the adult kidney at multiple AKI stages.

Previous work shows that Sox4 plays a crucial role in multiple developmental processes, including kidney, heart and immune system development (Hu and Chen, 2013; Huang et al., 2013; Paul et al., 2014; She and Yang, 2015). Sox4 was implicated in numerous human malignancies (Bilir et al., 2016; Tavazoie et al., 2008); moreover, the recent study reported that Sox4 promotes renal cell carcinoma metastasis via TGF $\beta$-induced EMT (Ruan et al., 2017). However, the role of Sox4 in AKI is unknown.

The scRNAseq data indicates the broad AKI induced Sox4 upregulation in multiple nephron tubule segments (Figure 2F, Table S1). Examining the pattern of Sox4 expression revealed a strong inverse relationship with SIc34a1-positive proximal tubules at UIR Day 4, when the injury starts resolving, as the combined feature plot (Figure 4A). Thus, we questioned whether Sox4 specifically marks the injured proximal tubules during the AKI response. To test this, we hybridized specific Sox4, SIc34a1 and Kim1 RNAscope probes to the injured and control kidney sections and found enhanced Sox4 expression in the Kim1-positive injured proximal tubules, exhibiting weakened SIc34a1 signal, at UIR Day 1 and 2 (Figure S4A). As previously described, UIR Day 4 and 7 exhibit substantially reduced Kim1 and restored Slc34a1 expression, consistent with kidney recovery. Notably, Sox4 was predominantly confined to remaining injured Kim1-positive proximal tubules at UIR Day 4 and 7 (Figures 4B, S4A), thus validating the scRNA-seq prediction. Quantitative IMARIS analysis revealed a substantial difference between Sox4 transcript number in the Kim1-enriched injured vs SIc34a1enriched mature proximal tubules (Figure S4B). Thus, we showed that Sox4 is a marker of proximal tubule dedifferentiation in adult AKI. 

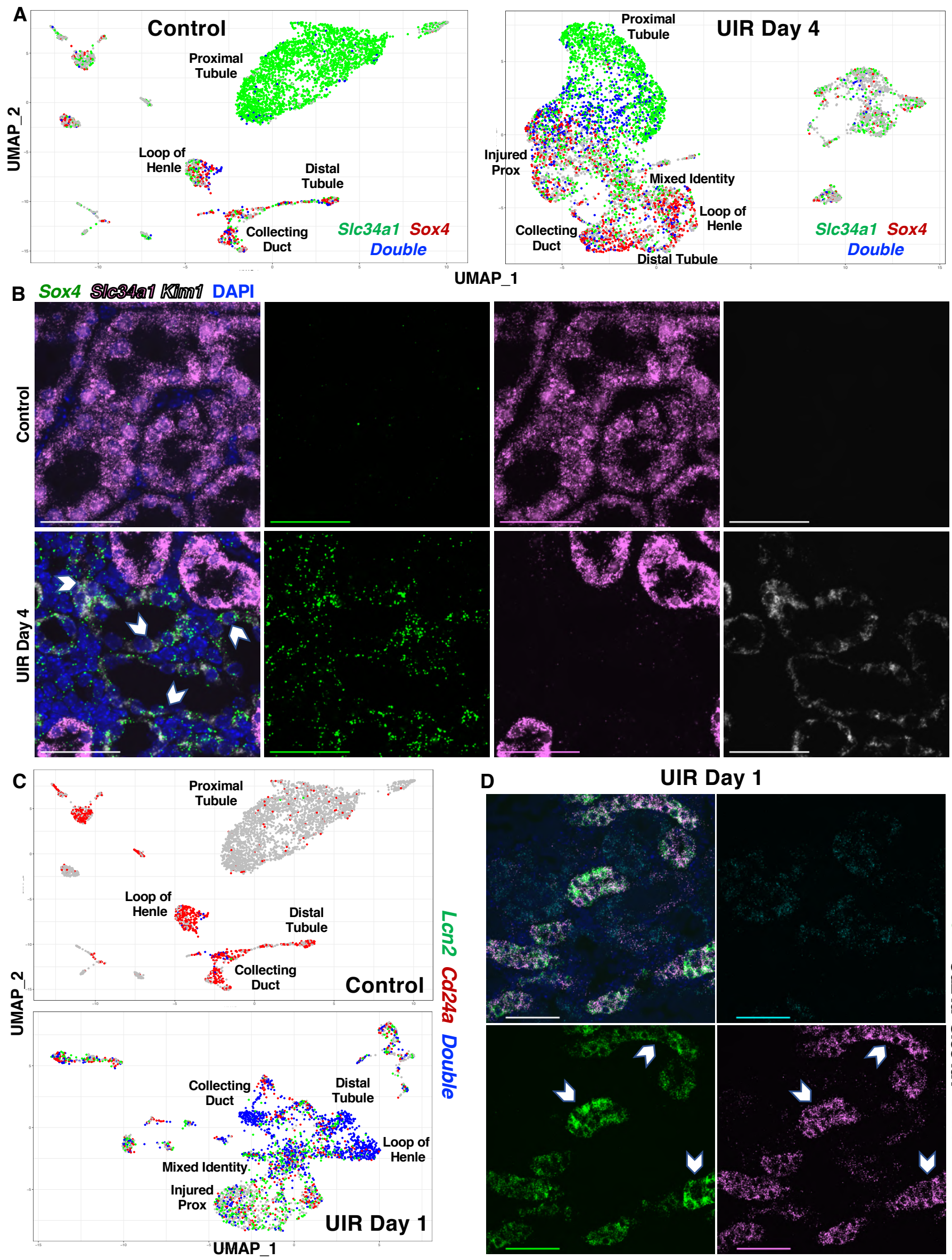

D

UIR Day 1

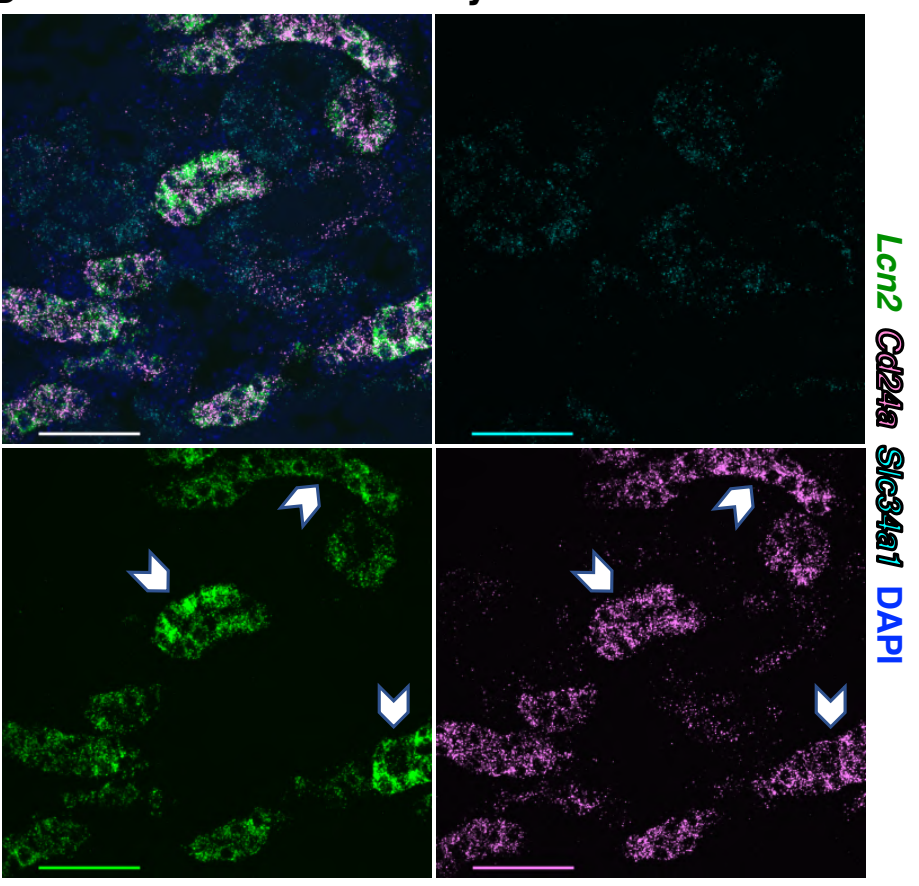




\section{Figure 4. Sox4 and Cd24a Label the Proximal and Distal Tubule Injury}

(A) Feature plots show SIc34a1 (green) and Sox4 (red) and double (blue), expression in UIR Day 4 vs Control.

(B) RNAscope with Slc34a1 (pink), Sox4 (green) and Kim1 (white) probes, DAPI (blue), UIR Day 4 vs Control. 60x Nyquist zoom, $0.14 \mu \mathrm{m} / \mathrm{px}$, Maximal Intensity Projection (MaxIP) from Z-stack, scale $50 \mu \mathrm{m}$.

(C) Feature plots show Lcn2 (green), Cd24a (red) and Double (blue) positive cells in UIR Day 1 vs Control.

(D) 60x RNAscope images show Lcn2 (green) and Cd24a (pink) colocalization at UIR Day 1, 60x Nyquist zoom, 0.21 $\mu \mathrm{m} / \mathrm{px}$, Maximal Intensity Projection (MaxIP) from Z-stack, scale $50 \mu \mathrm{m}$.

Related to Figures S4 and S5.

Next, we assessed whether renal developmental genes mark renal injury in the distal nephron tubule segments. We observed that $C d 24 a$ notably overlaps with Lipocalin 2 (Lcn2), a clinically recognized distal marker (Devarajan, 2008, 2010; Mishra et al., 2005) used in clinical practice as an early predictor and progression marker of AKI (Khawaja et al., 2019; Sun et al., 2018; Zhang et al., 2017a). As the combination UMAPs show, the injured kidney exhibits a prominent number of double Cd24a; Lcn2 positive cells in the loop of Henle, distal tubule, collecting duct and "Mixed Identity Cells" at UIR Day 1 (Figure 4C). We detected the highest Lcn2 and Cd24a levels at the most severe stages of kidney injury (UIR Day 1 and 2), which returned back to normal by Day 14 (Figures 3F, S3B, S3C). Examining the spatial pattern of Cd24a and Lcn2 expression with RNAscope revealed their striking level of colocalization at multiple time points after AKI (Figure 4D, S5A).

The scRNAseq data also showed that Cd24a increases in the proximal tubules in response to the injury, as discussed earlier. To quantitatively assess the Cd24a expression in the different injured kidney compartments, we counted Cd24a within Slc34a1-positive proximal tubules vs Lcn2-enriched injured tubules using IMARIS. We found that UIR caused Cd24a increase in SIc34a1-positive proximal tubules at Day 1, 2 and 4, however, it was substantially more enriched in the Lcn2-positive injured compartment (Figures S5B, S5C). Moreover, we found that Cd24a and Lcn2 transcript numbers exhibit remarkable positive correlation, as the Pearson analysis shows (Figure S5D). 
Importantly, AKI induced Cd24a transcriptional changes were reproduced on the protein level, as we show with Cd24a immunohistochemistry (IHC) (Figure S5E). Thus, we demonstrate that renal developmental genes might serve as new AKI markers.

\section{Novel Gene Expression Signatures of AKI}

With scRNA-seq, we found that AKI induced some global gene expression changes in the kidney, in multiple compartments, exemplified by the cytokine and Clusterin signaling discussed earlier. We also found the widespread Spp1 (a.k.a. Osteopontin) upregulation caused by AKI, including in tubular, stromal and immune cells (Figures 5A and 5B). Spp1 is a secreted phosphoprotein 1 which plays a major role in bone metabolism and immune system activation, particularly, macrophages and T- and B-cell mediated immunity; Spp1 is implicated in multiple renal pathologies, including renal cell carcinoma, diabetic nephropathy and allograft rejection (Kaleta, 2019). Consistent with the previous work, we observed that Spp1 exhibits some baseline expression mostly in the renal medulla; however, UIR caused striking Spp1 elevation in both cortex and medulla (Figure 5A). We observed that Spp1 expression overlaps with another kidney injury associated gene, Krt8, in the injured nephron tubules and collecting duct (Figure 5B). Elevated cytokeratin tubular expression is a known characteristic of epithelial cell stress (Djudjaj et al., 2016; Liu et al., 2017); consistent with these observations, we identified Krt7, 8 and 18 elevation in the UIR injured tubules (Table S1). Notably, aberrant cytokeratin expression is associated with tumor progression and metastasis (Fang et al., 2017). In particular, Krt8 was shown to play a crucial role in renal cell carcinoma (Tan et al., 2017). Immunofluorescence (IF) showed that $K r t 8$ transcript elevation was accompanied by increased protein (Figure S6A); moreover, we identified Krt8-positive renal tubules which also expressed Spp1, using a combined $\mathrm{CISH}$ and IF approach (Figure 5C). We observed that Krt8 and Spp1 levels remained elevated through UIR Day 4 and lowered to the normal levels by Day 14, indicating injury 
A
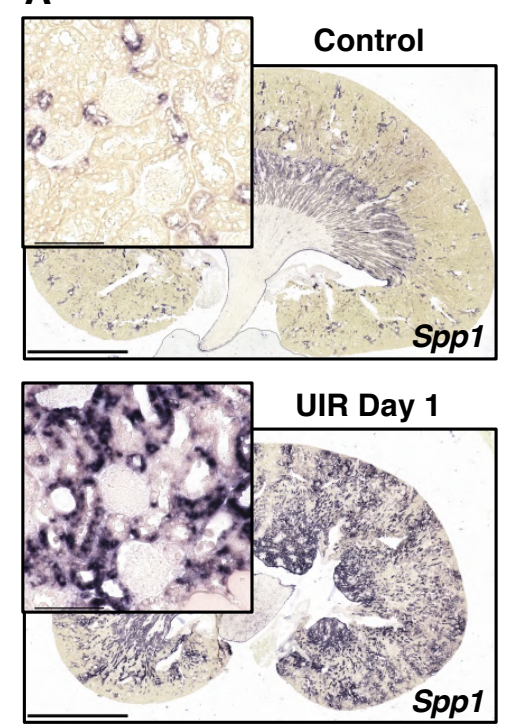

D

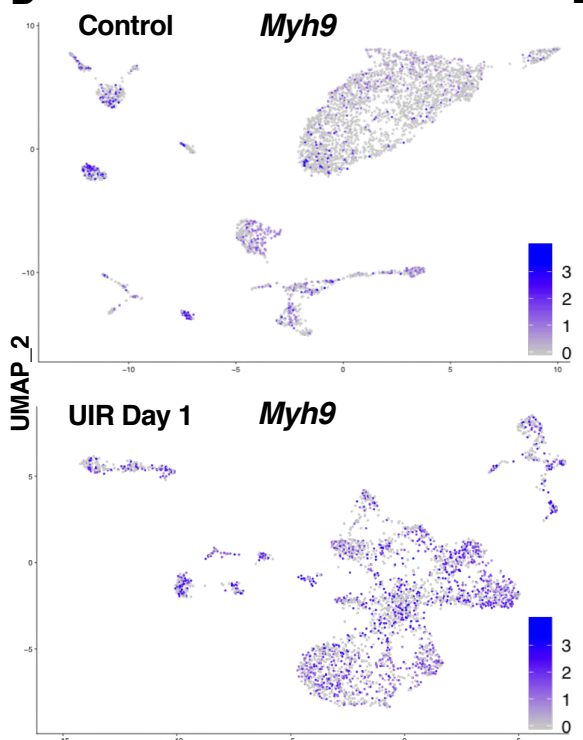

UMAP_1

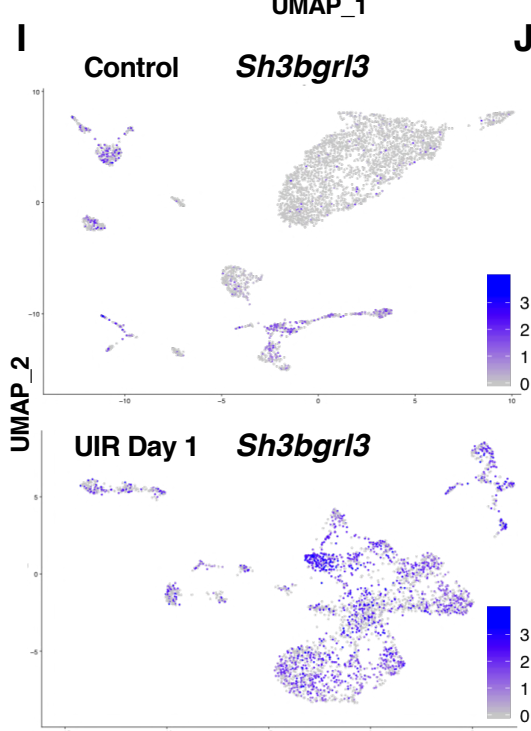

UMAP_1
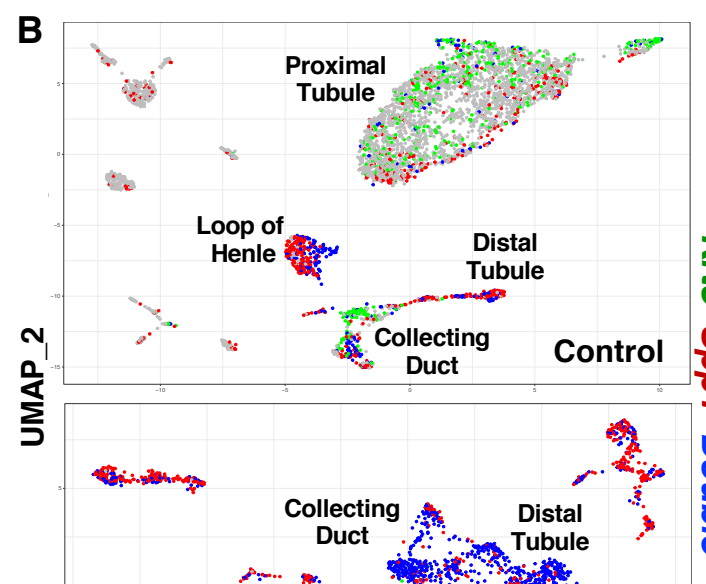

Mixed Identity

Injured Prox UMAP 1
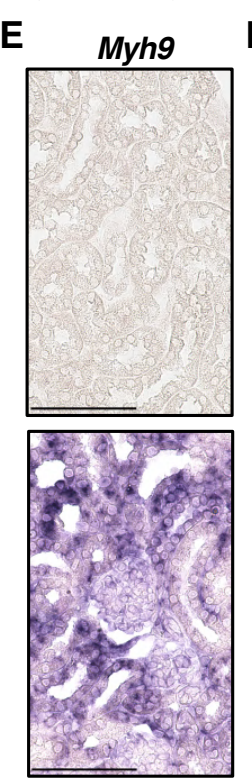

Sh3bgrl3

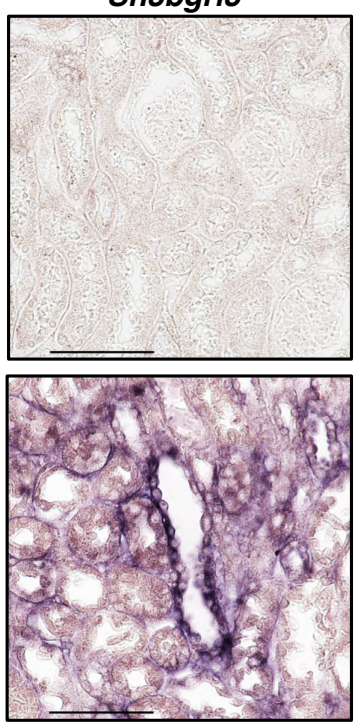

$\sum_{5}^{\complement}$ UIR Day 1 Ahnak
F.
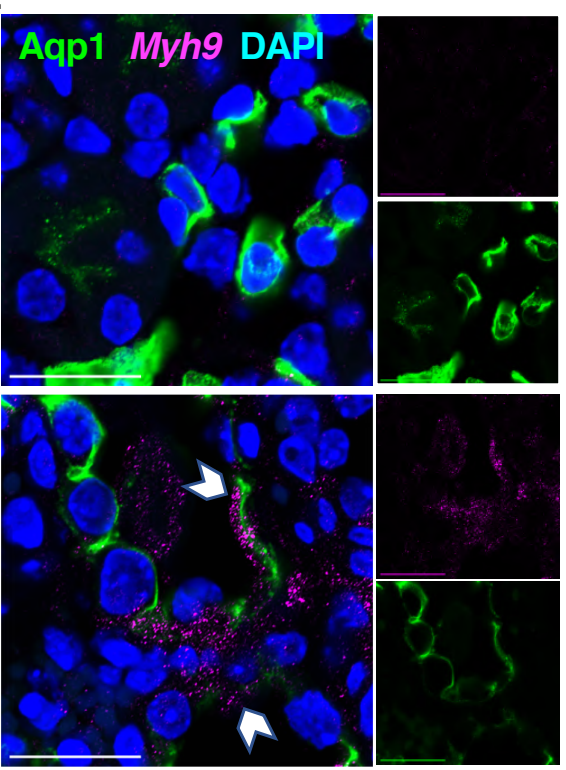

K

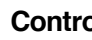

Ahnak
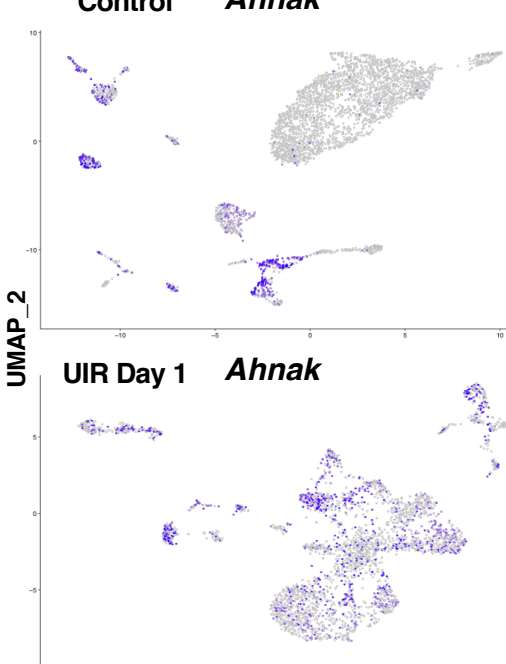

UMAP_1

\section{Krt8 Spp1 DAPI}

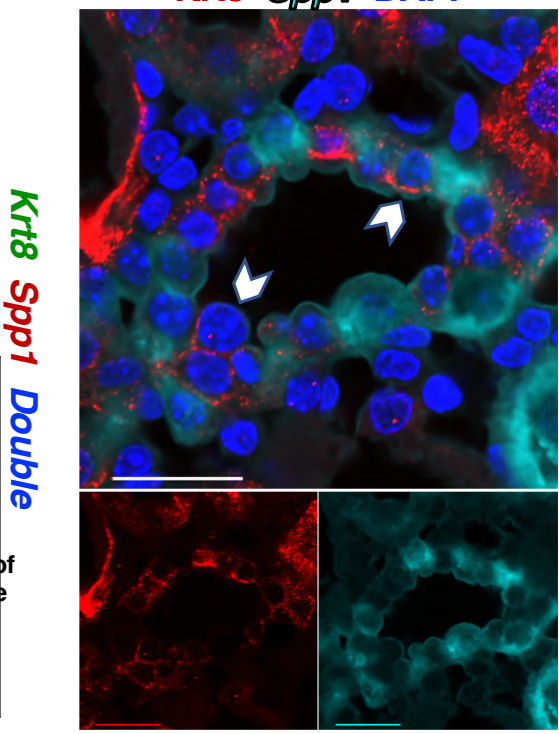

G
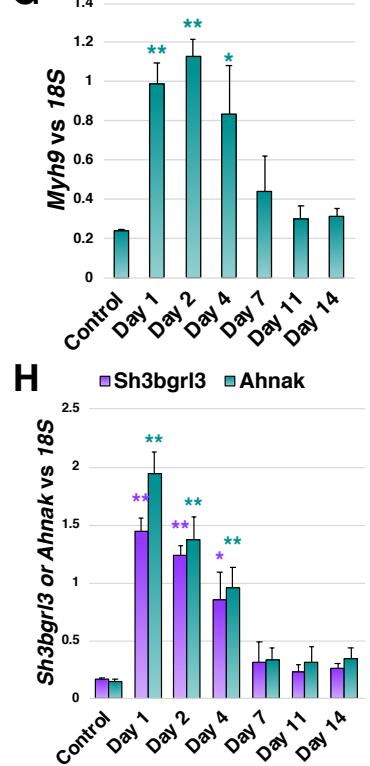

$\mathbf{L}$
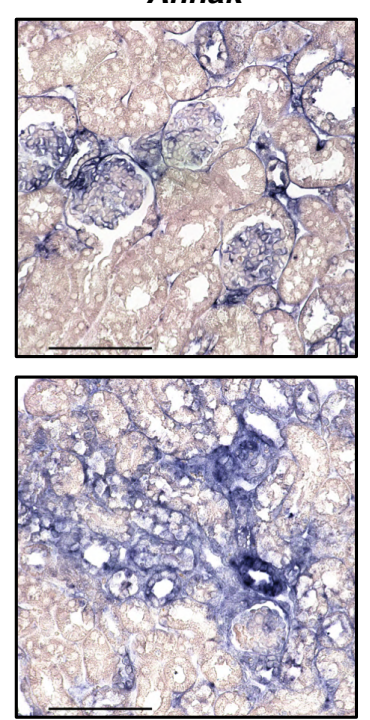


\section{Figure 5. Novel Gene Expression Signatures of AKI}

(A) Spp1 CISH, Control vs UIR Day 1, 4x and 40x zoom into the cortex.

(B) Combined Feature plots show Krt8 (green), Spp1 (red) and Double (blue) positive cells in UIR Day 1 vs Control.

(C) Spp1 CISH (cyan), Krt8 IF (red), DAPI (blue), UIR Day 1, 60x Nyquist 3x zoom. Pointers show Spp1 and Krt8 overlap. The combined scRNA-seq and CISH results define the precise cell types with elevated Spp1 and Krt8 elevated expression following UIR.

(D) Feature plots show Myh9 expression in UIR Day 1 vs Control.

(E) Myh9 CISH shows elevated expression in UIR Day 1 tubules, glomeruli and interstitium compared to the Control, 40x.

(F) Myh9 CISH (magenta), Aqp1 IF (green), DAPI (blue), Control vs UIR Day 1, 60x Nyquist 3x zoom. Pointers show Myh9 within Aqp1-positive tubules.

(G and H) qPCR shows Myh9, Sh3bgrl3 and Ahnak expression over AKI course, Mean + SEM, 4-6 animals per group, one way ANOVA with Bonferroni and Holm, ${ }^{*} p<0.05,{ }^{* *} p<0.01$ compared to the Control.

(I) Feature plots how Sh3bgrl3 expression in Control vs UIR Day 1.

(J) Sh3bgrl3 CISH shows elevated expression in UIR Day 1 tubules and interstitium compared to the Control, 40x.

(K) Feature plots show Ahnak expression in Control vs UIR Day 1.

(L) Ahnak $\mathrm{CISH}$ shows expression in the glomeruli and interstitium of the Control, and elevated expression in UIR Day 1 tubules and interstitium, 40x.

Scale 4x, 2500um; 40x, 100um; 60x Nyquist 3x zoom, 25 $\mu \mathrm{m}$.

Related to Figure S6.

resolution (Figure S6A). Overall the results show elevation of multiple established kidney epithelial injury markers and reveal the novel AKI induced overlapping Spp1 and Krt8 expression. Since both Spp1 and Krt8 play a role in renal cell carcinoma metastasis and progression, dual Spp1-and Krt8positive cells give evidence for renal tubular cells acquiring a more mesenchymal phenotype in response to the injury.

scRNAseq also identified the elevation of Myosin Heavy Chain 9, or Myh9, a non-muscle myosin involved in cell motility, cell shape maintenance and cytokinesis, in the injured renal tubules. As we and the others (Arrondel et al., 2002) show, normal adult kidney mostly expresses Myh9 in the stromal cells, pericytes, glomeruli and endothelial cells, with very faint tubular expression (Figure 5D). As both scRNAseq and $\mathrm{CISH}$ indicated, UIR caused a marked Myh9 elevation in all renal tubule compartments, including the sub-clusters of injured and dedifferentiated proximal tubules, and in the 
collecting ducts (Figures 5D and 5E). Importantly, previous work specifically reported that Myh9 expression is absent in normal proximal tubules (Otterpohl et al., 2017). We validated the injured proximal tubule expression via co-staining the Myh9 CISH with Aqp1 IF and identified Aqp1-positive proximal tubules exhibiting elevated Myh9 expression which was not detected in the control (Figure $5 F)$, providing an additional evidence for the injury induced mesenchymal phenotype in renal epithelial tubules. With qPCR, we show that Myh9 elevation persists to UIR Day 4 and then reverses to the normal levels (Figure 5G).

The scRNAseq approach allowed us to identify additional novel genes not previously implicated in AKI. For examples, we found that Sh3bgrl3 and Ahnak were upregulated in the UIR treated kidneys, as validated by $\mathrm{qPCR}$ (Figure $5 \mathrm{H}$ ). Sh3bgrl3 encodes the SH3 Domain-Binding Protein 1, associated with GTPase, oxidoreductase and anti-apoptotic activity (Henn et al., 2001). The control kidney exhibited very low levels of Sh3bgrl3, while it was highly enriched in all tubular lineages in UIR Day 1 (Figure 5I). CISH confirmed the AKI induced Sh3bgrl3 elevation within renal tubules and in peritubular spaces (Figure 5J). The other gene, Ahnak, encodes Neuroblast Differentiation-Associated Protein AHNAK, involved in pathogenesis of Miyoshi Muscular Dystrophy (Cacciottolo et al., 2011) and tumor metastasis via TGF- $\beta$ induced epithelial-to-mesenchymal transition (Sohn et al., 2018). Notably, both scRNAseq and $\mathrm{CISH}$ showed that Ahnak is present in the Control podocytes and stroma with some tubular expression (Figures 5K and 5L), however, UIR resulted in the marked Ahnak elevation in the tubular and stromal compartments. With qPCR, we showed that both Ahnak and Sh3bgrl3 stay elevated until UIR Day 4, and then return to normal expression levels (Figures 5G, 5H). As the scRNAseq data shows, both Ahnak and Sh3bgrl3 are highly enriched in the injured proximal tubules while nearly absent in the healthy ones, suggesting they represent novel proximal tubule injury markers. 


\section{scRNAseq Identifies Novel Epithelial-to-Stromal Interactions in the Adult AKI}

Growing evidence suggests the importance of cell-to-cell interactions in kidney injury progression (Humphreys, 2018). Thus, we examined the molecular nature of normal and pathologic intracellular interactions in the kidney. To this end we paired the cells enriched with ligand to the cells enriched for the corresponding receptor, and found, for examples, that the proximal and distal tubules and the stromal cells might interact with each other via the calmodulin (Calm1, Calm2, Calm3), growth factor (Egf, Fgf1, Hbegf, Vegfa, Mdk, Igfbp4) and lipid metabolism (Lpl, Lrpap1, Psap) pathways in the normal kidney (Figure 6A, Table S4). We also found that the stromal cells might influence proximal tubules via collagen signaling, since the stromal cells were enriched with Col1a1/1a2, Col3a1, Col4a1/4a5 and Col18a1, while the proximal tubules expressed their receptors. Our data also highlights potential pericyte-to-proximal tubule interactions via the collagen, nidogen (Nid1, Nid2), matrix metalloproteinase (Timp2) and calmodulin signaling.

Next, we examined how AKI affects cell-to-cell communication in the kidney and focused on UIR Day 4, as an intermediate injury response time point. Ligand-to-receptor analysis revealed that AKI causes a dramatic increase in the interactions between the renal cell populations. In particular there was elevated extracellular matrix signaling by the injured epithelial cells (Figure 6B, Table S4). We observed the elevation of Vimentin (Vim), which is known as an activated fibroblasts marker associated with EMT (Liu et al., 2015; Liu, 2010) and kidney fibrosis (Grande et al., 2015; Humphreys, 2018), in the injured proximal tubules and mixed identity cells (Figure S6B). Moreover, we found that the Vim receptor encoding gene Cd44 (Pall et al., 2011) was expressed in the stromal cells, suggesting the pathologic injury induced tubular-to-stromal crosstalk. Importantly, we noticed that $C d 44$ was also enriched in the injured proximal tubules and in the mixed identity population 
themselves, suggesting that injured renal epithelial cells not only interact with stromal cells, but crosstalk with each other in the injury setting.

Of particular interest, we also observed that AKI induced elevation of Col18a1, encoding an extracellular nonfibrillar collagen which is a necessary component of basement membrane (Kinnunen et al., 2011). We and others (Harding et al., 2011; McMahon et al., 2008) show that Col18a1 is normally present mostly in the glomerulus with some weak expression in the collecting duct (Figure $\mathrm{S} 6 \mathrm{~B})$, however, its role in the $\mathrm{AKI}$ response remains unknown. We found remarkable Col18a1 elevation in the injured proximal and distal tubules and the mixed identity cells caused by AKI; moreover, our analysis identified Col18a1 receptors encoding genes Gpc4 and Itgb1 to be enriched in the stromal cells, which highlights another potential epithelial-to-stromal cell interaction pathway (Figure 6B, Table S4). These findings were validated by the qPCR, which showed significant Vim and Col18a1 elevation caused by AKI (Figures S6C-S6D). Moreover, RNAscope revealed the marked Vim and Col18a1 upregulation within the Slc34a1-positive proximal tubules at UIR Day 1, 2 and 4, which returned to the normal interstitial and periglomerular expression pattern by Day 14 (Figures 6C and 6D, S6E, Movies S5 and S6). Other factors involved in injury induced epithelial-to-stromal crosstalk included collagen (Col4a1, Col4a3, Col4a4), lipocalin 2 (Lcn2), interferon (Ifitm2), galectin (Lgals3bp) and Osteopontin (Spp1) (Table S4). Thus, our scRNAseq experiments revealed that renal epithelial and stromal cells show increased potential interactions with each other in the condition of injury. 
A

B

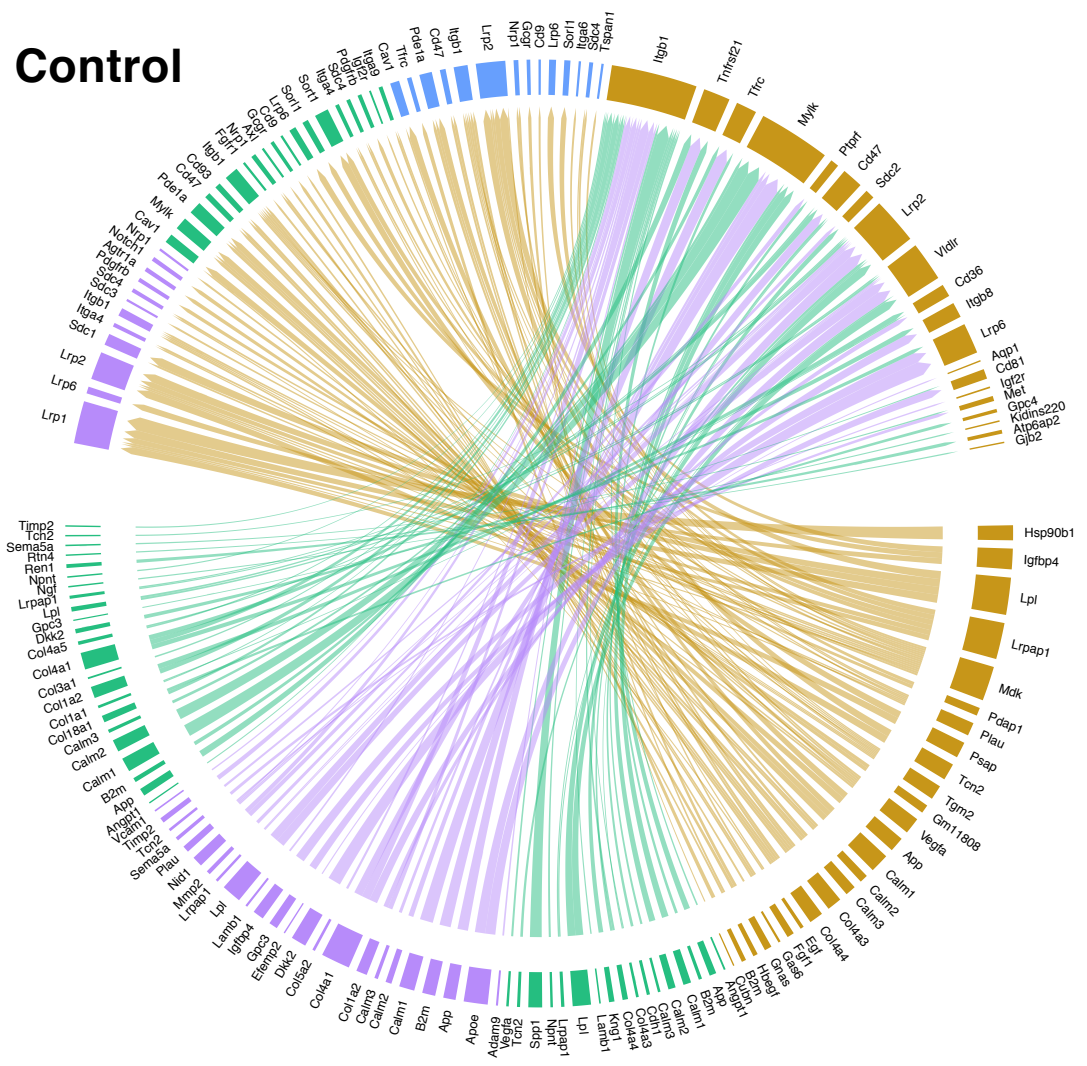

\section{UIR Day 4}

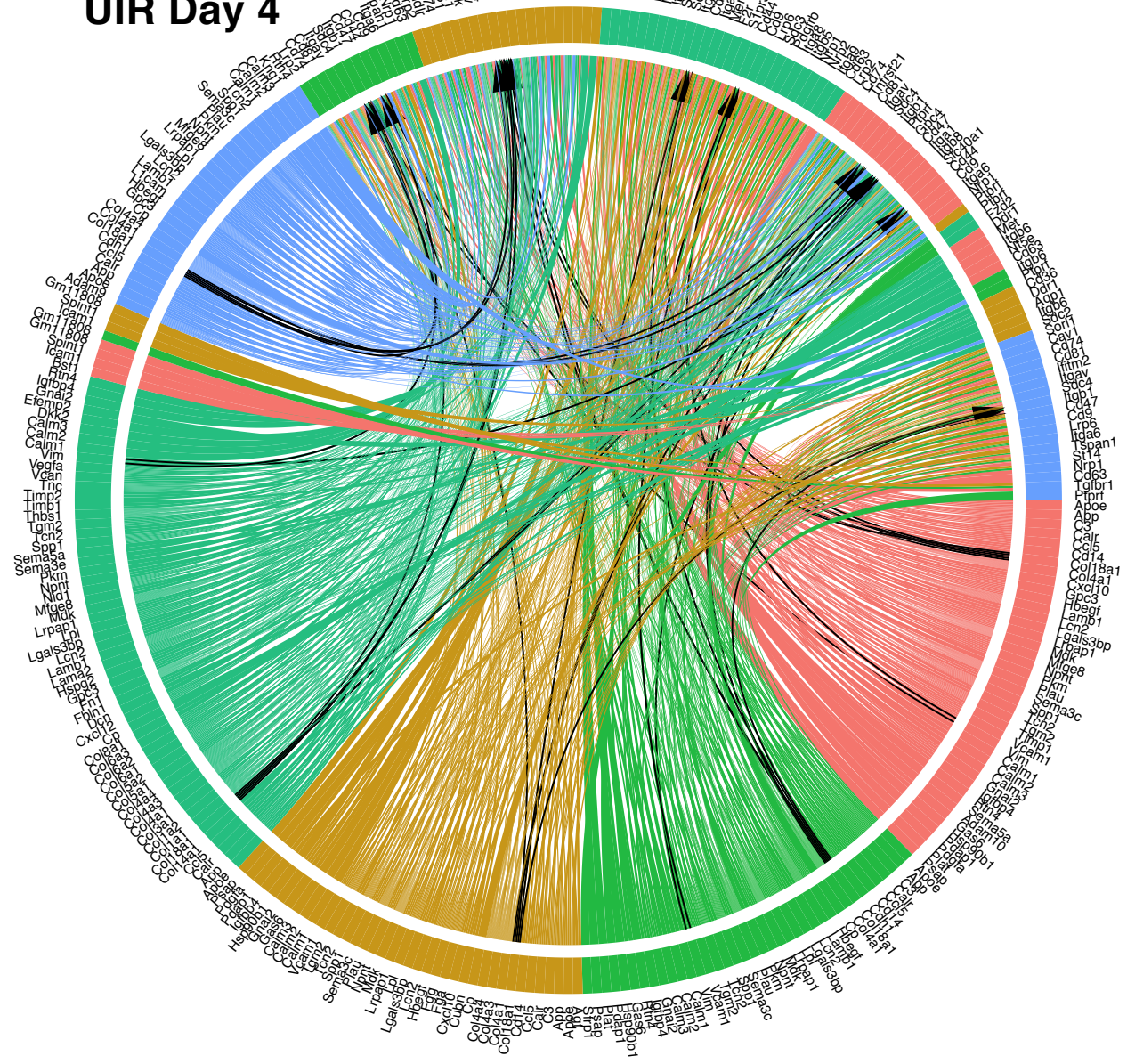

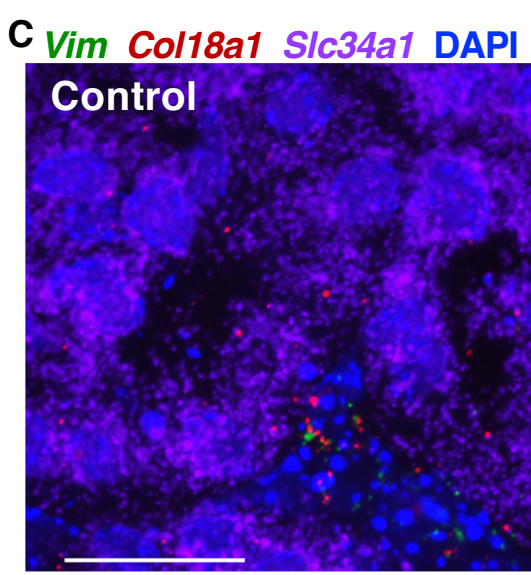
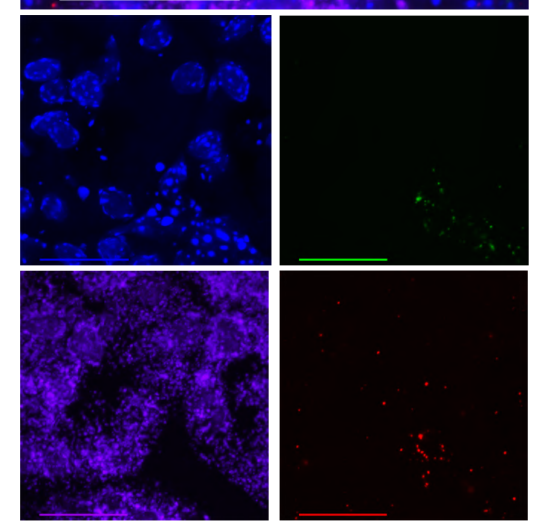

D
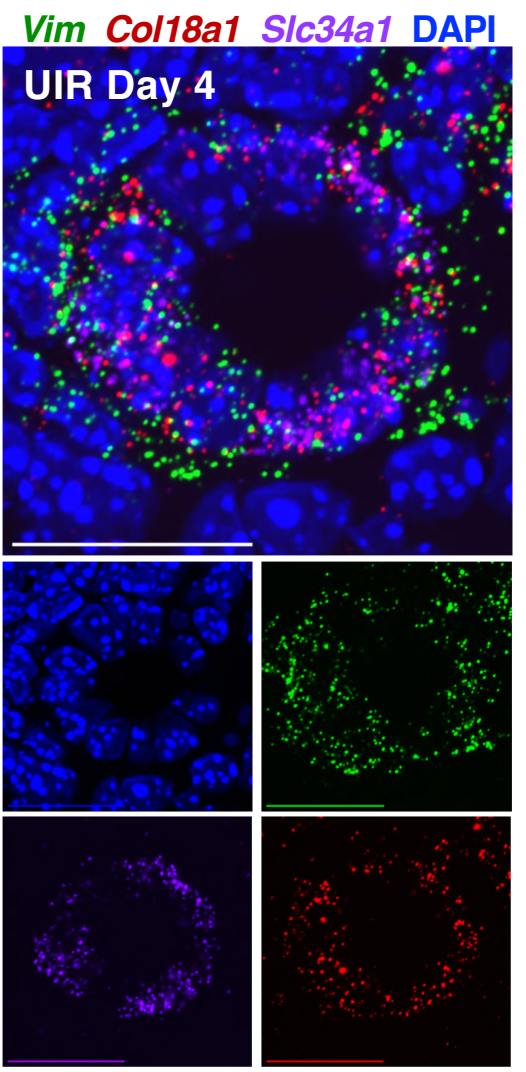


\section{Figure 6. scRNAseq Identifies Novel Epithelial-to-Stromal Interactions in the Adult AKI}

(A) Circos Plot of ligand-receptor interactions between the proximal (brown) and distal (blue) tubules, the stromal cells (green), and the stromal/pericyte cells (purple) in the normal kidney.

(B) Circos Plot of ligand-receptor interactions between the proximal tubules (brown), injured prox (red), mixed identity cells (green), distal tubules (blue) and the stromal cells (teal) in the normal kidney. Black arrows show Vim-Cd44, Col18a1Gpc4 and Col18a1-Itgb1 Ligand-Receptor pairs. Note the dramatic increase in the number of potential interactions compared to control, using the same filters.

(C) Representative RNAscope image of Control proximal tubules, Vim (green), Col18a1 (red), Slc34a1 (purple), 60x 4x Nyquist zoom, MaxIP from the Z-stack.

(D) Representative RNAscope image of UIR Day 4 proximal tubule, Vim (green), Col18a1 (red), Slc34a1 (purple). 60x 4x Nyquist zoom, MaxIP from the Z-stack.

Scale $50 \mu \mathrm{m}$.

Related to Figure S6, Table S4, Movie S6 and S7.

\section{Renal Developmental Genes Outline Kidney Fibrotic Remodeling}

Next, we tested if the AKI induced genes identified by the scRNAseq could serve as markers of maladaptive injury response and fibrotic remodeling. We induced the identical UIR in 10 weeks old male Swiss-Webster mice (Figure 7A) and found that they exhibited significantly elevated fibrosis marker expression, prominent fibrotic remodeling and decreased mature proximal tubule marker SIc34a1 expression at UIR Day 14 compared to the younger mice (Figures 7B-7D). Thus, we show that age of $\mathrm{AKI}$ onset is a definitive factor in determining whether the kidney undergoes repair or maladaptive remodeling. Notably, we observed that both Sox4 and Cd24a were significantly elevated for longer times in the older animals compared to the younger ones (Figures 7E, 7F). Moreover, IHC showed that Cd24a stays elevated in renal tubules of the older mice throughout the AKI course, while it resolves in the younger animals by UIR Day 14 (Figure 7G). This shows that AKI induced Cd24a transcriptional increase also occurs at the protein level. Overall, our data reveals that renal developmental genes outline AKI progression and might serve as predictors of the injury outcome. 
A

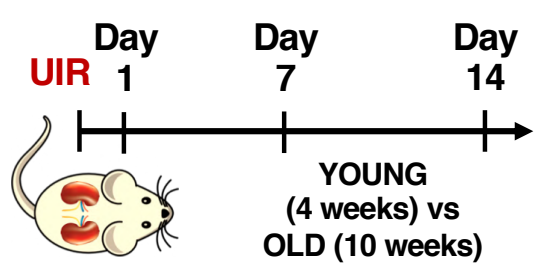

B
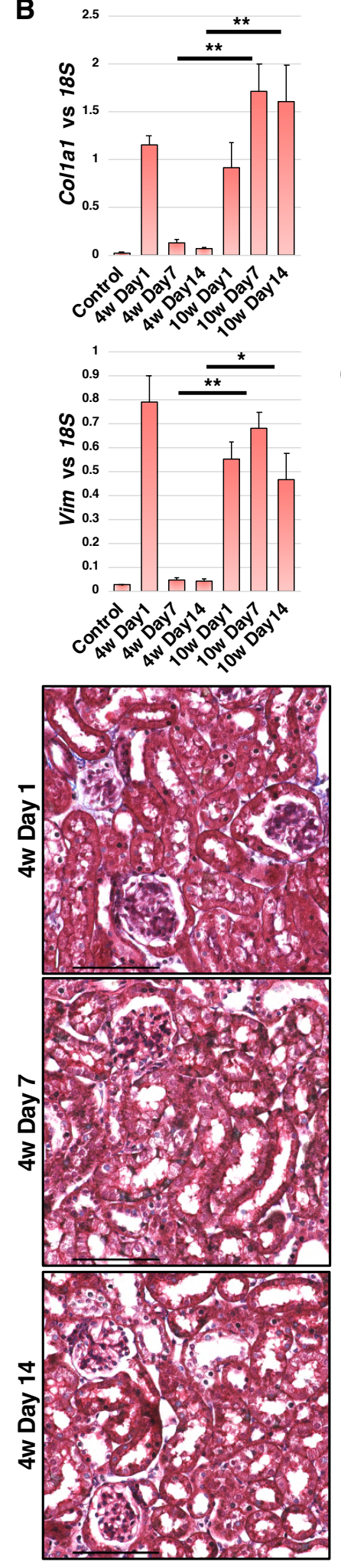

E $^{1.5}$
D
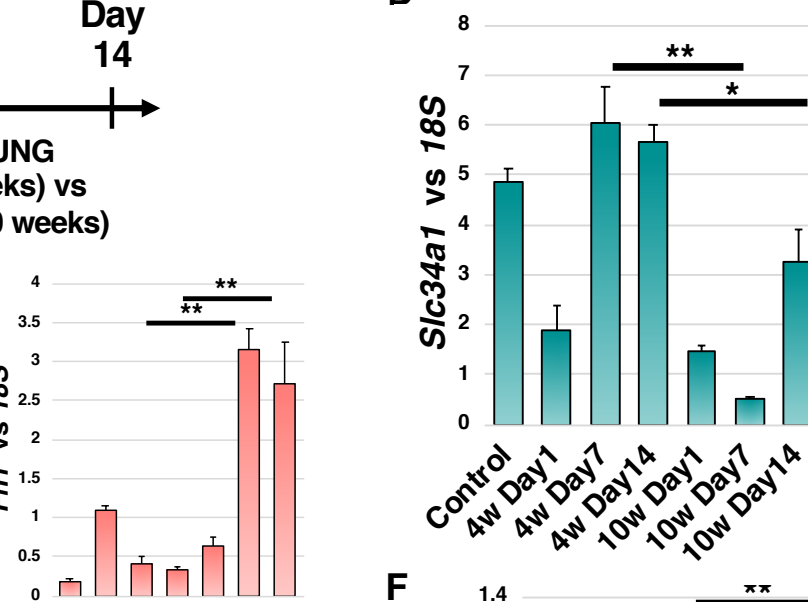

cond

\section{Masson's Trichrome}
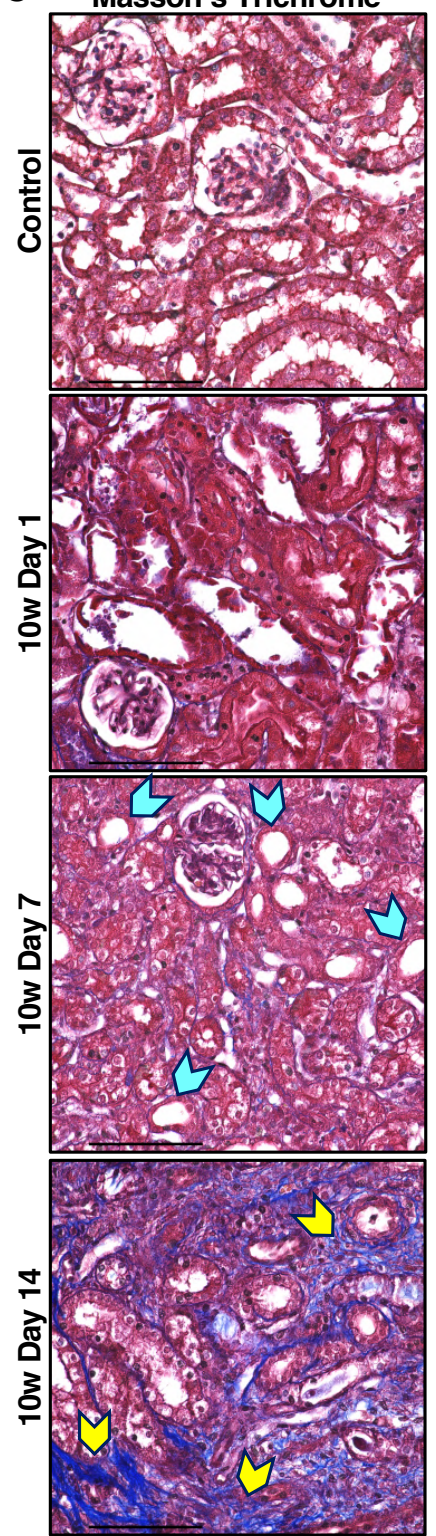

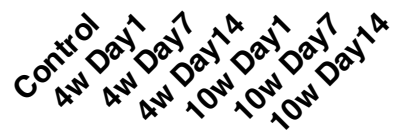

$\mathbf{F}$
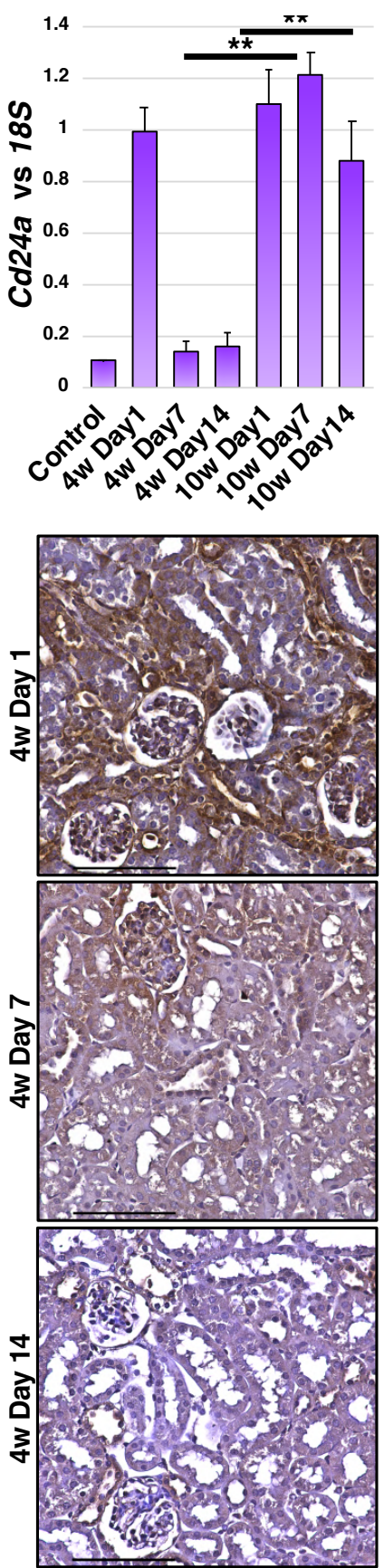

E

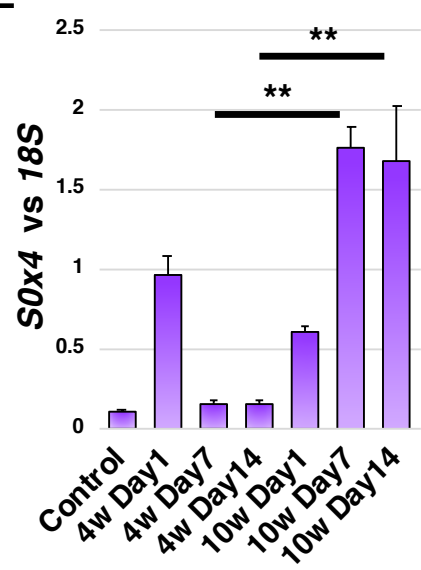

G

Cd24a

Immunohistochemistry
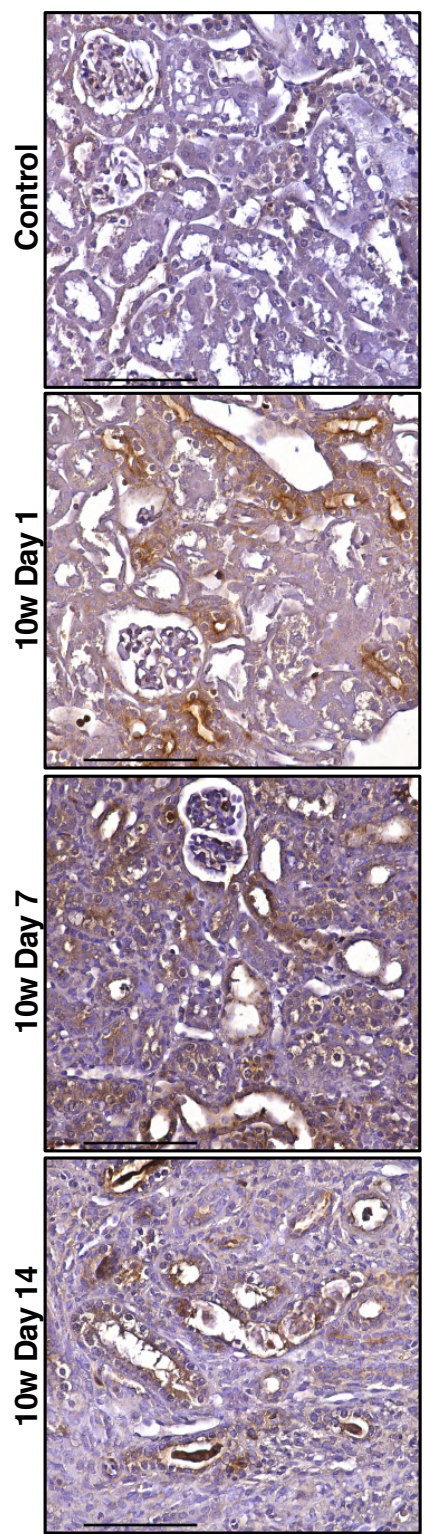


\section{Figure 7. Renal Developmental Genes are Elevated in Kidney Fibrotic Remodeling}

(A) Experimental outline.

(B) qPCR shows the fibrosis markers Vim, Col1a1 and Fn1 expression over the AKI course, Mean \pm SEM, $\mathrm{n}=4-6$ per group, Student's $t$ test, $4 \mathrm{w}$ Day 7 vs $10 \mathrm{w}$ Day7, 4w Day 14 vs $10 \mathrm{w}$ Day $14,{ }^{*} \mathrm{p}<0.05,{ }^{* *} \mathrm{p}<0.01$.

(C) Masson's Trichrome staining shows fibrotic remodeling in the $10 \mathrm{w}$ old mice at UIR Day 14; tubular dilation - blue pointers, fibrotic areas - yellow pointers; 40x.

(D-F) qPCR shows Slc34a1, Sox4 and Cd24a expression over the AKI course, Mean \pm SEM, $n=4-6$ per group, Student's $t$ test, Day $74 \mathrm{w}$ vs $10 \mathrm{w}$, Day $144 \mathrm{w}$ vs $10 \mathrm{w},{ }^{*} \mathrm{p}<0.05,{ }^{* *} \mathrm{p}<0.01$.

(G) Cd24a IHC, 40x.

Scale $100 \mu \mathrm{m}$.

\section{Acknowledgements}

The authors thank members of Jiang and Yutzey laboratories for chromogenic in situ hybridization and RNAscope protocols. The authors thank members of Cincinnati Children's Hospital Medical Center Gene Expression Core, Confocal Imaging Core and Veterinary Services Surgical Core for valuable guidance and assistance with imaging, scRNA sequencing and animal procedures. This work was supported by NIH grant DK120842 (SSP), Mallinckrodt Fellowship Award (KAD).

\section{Author Contributions}

V.R-M., M.A. and S.S.P. prepared the manuscript. V.R-M. assembled figures. A.P. did kidney dissociation and scRNA-seq. M.A. analyzed the scRNA-seq data. Q.M. and V.R-M. induced UIR. V.R., M.A., P.D. and S.S.P. developed experimental strategy and analyzed results. V.R-M., S.M.C. and Q.M. did validation experiments. M.P.S. and J.M.K. designed imaging configurations and provided guidance for RNAscope data analysis. K.A.D. helped to cover the sequencing costs.

\section{Declaration of Interests}

The authors declare no competing interests. 


\section{Methods}

\section{Lead Contact and Materials Availability}

Further information and requests for resources and reagents should be directed to and will be fulfilled by the Lead Contact, Dr. S. Steven Potter (Steve.Potter@cchmc.org).

\section{Experimental Model and Subject Details}

The Institutional Care and Use Committee (IACUC) of Cincinnati Children's Hospital Medical Center reviewed and approved all animal procedures used in this study. The unilateral ischemia/reperfusion (UIR) (Le Clef et al., 2016; Yang et al., 2010) was induced via atraumatic left renal pedicle clamping for $30 \mathrm{~min}$ at $37^{\circ} \mathrm{C}$ in the 4 weeks old male Swiss-Webster mice, and the kidneys were harvested at Day $1,2,4,7,11$ and 14 after the UIR ( $n=1$ per each time point) for the single cell RNA sequencing (scRNAseq) and histological verification. Kidneys harvested from the naïve mice of the same strain and age were used as controls. The validation was performed on the additional set of animals treated with the identical UIR procedure ( $n=3-6$ per group). For examining the effects of age on the AKI outcome, the equivalent UIR was induced in the 10 weeks old male Swiss-Webster mice, and the kidneys were harvested at UIR Day 1, 7 and 14 ( $n=4$ per group). All animal procedures were in concordance with the institutional protocols.

\section{Method Details}

\section{Experimental Animals and AKI Procedure}


All animal procedures were conducted according to the Institutional Care and Use Committees

(IACUC) of Cincinnati Children's Hospital Medical Center guidelines. Swiss-Webster (CFW) 4 and 10 weeks old male mice obtained from Charles River Laboratories were subjected to the UIR model of acute kidney injury $(\mathrm{AKI})$. The animals were anesthetized with isoflurane and placed on the homeothermic blanket, and the body temperature was maintained at $37^{\circ} \mathrm{C}$. Ischemia was induced via the midline abdominal approach with the atraumatic vascular clamp placed on the left kidney pedicle and verified with the immediate kidney color change (Skrypnyk et al., 2013), with the right kidney left undisturbed. The clamp was released after $30 \mathrm{~min}$ followed by the left kidney color change back to red. The animals were closed via abdominal muscle and skin suturing, given $1 \mathrm{ml}$ of warm sterile normal saline subcutaneously for the fluid loss compensation and returned to the normal housing. The animals received $50 \mathrm{ml}$ of $0.03 \mathrm{mg} / \mathrm{ml}$ Buprenorphine subcutaneously for analgesia on the day of surgery and on the next day, and were monitored daily according to the IACUC requirements.

\section{Single Cell Suspension Preparation and scRNA-seq procedure}

The 4 weeks old male CFW mice treated with the UIR procedure were intraperitoneally injected with $100 \mu \mathrm{L}$ heparin $(100 \mathrm{U} / \mathrm{mL})$, anesthetized with isoflurane chamber and euthanized via exsanguination followed by cervical dislocation at Day $1,2,4,7,11$ and 14 after the UIR ( $n=1$ per group). The kidneys were perfused with ice-cold DPBS via the aorta prior to the harvesting to remove the red blood cells, and the left (injured) and the right (contralateral) kidneys from the UIR treated mice were collected along with the control kidneys obtained from the naïve mice. The kidneys were placed on ice-cold Dulbecco's phosphate-buffered saline (DPBS), decapsulated, bisected lengthwise and sliced coronally. The cortical regions were then finely minced with a sterile razor blade until tissue was homogenous and clumps were broken down, and $65 \mathrm{mg}$ of the minced tissue was placed in $2 \mathrm{ml}$ 
digestion buffer containing $3 \mathrm{mg} / \mathrm{mL}$ Type 2 collagenase (Worthington, Collagenase Type 2), 1.5 $\mathrm{mg} / \mathrm{mL}$ ProNase E (Sigma P6911), $62.5 \mathrm{U} / \mathrm{mL}$ DNAse (Applichem, A3778), and $5 \mathrm{mM} \mathrm{CaCl} 2$ made up in DPBS. The digest mix was incubated in a $37^{\circ} \mathrm{C}$ water bath for 20 minutes with vigorous trituration with a $1 \mathrm{~mL}$ pipet every $2 \mathrm{~min} .5 \mu \mathrm{L}$ aliquots were taken every $5 \mathrm{~min}$ and visualized using scope to ensure adequate digestion. After $20 \mathrm{~min}$, the digest mix was added to two $1.5 \mathrm{~mL}$ low-adhesion tubes and was then incubated in a thermomixer for $5 \mathrm{~min}$ at $37^{\circ} \mathrm{C}$ while shaking at $1400 \mathrm{RPM}$. The digest mix was subsequently added to a $70 \mu \mathrm{M}$ filter (Miltenyi, 130-098-462) stacked on a $30 \mu \mathrm{M}$ filter (Miltenyi, 130-098-458) on a $50 \mathrm{~mL}$ conical tube. The filters were rinsed with $10 \mathrm{~mL}$ PBS/BSA $0.01 \%$ and the flow-through was then transferred to a $15 \mathrm{~mL}$ conical tube. The cells were pelleted by centrifugation at $350 \mathrm{~g}$ for $5 \mathrm{~min}$ at $4{ }^{\circ} \mathrm{C}$. The supernatant was discarded, and the pellet re-suspended in $100 \mu \mathrm{L}$ PBS/BSA. The cells were visualized and if $>30 \%$ were RBC, RBC lysis step was performed: $1 \mathrm{~mL}$ of RBC lysis buffer (Sigma, R7757) was added, and the cells were triturated 20x with a $1 \mathrm{~mL}$ pipet; the cells were then incubated for $2 \mathrm{~min}$ on ice. $12 \mathrm{~mL}$ ice-cold PBS/BSA was then added to dilute the RBC lysis buffer. If no RBC lysis was necessary, then $12 \mathrm{~mL}$ ice-cold PBS/BSA was added to the cells. The cells were pelleted by centrifugation at $350 \mathrm{~g}$ for $5 \mathrm{~min}$ at $4{ }^{\circ} \mathrm{C}$. The supernatant was then discarded, and the cells re-suspended in $2 \mathrm{~mL}$ PBS/BSA. Cells were analyzed with a hemocytometer using trypan blue and the concentration was adjusted to $100,000 \mathrm{cells} / \mathrm{mL}$ for the droplet-based single-cell RNA-seq procedure (DropSeq) based on a protocol from Macosko et. al (Macosko et al., 2015).

The remaining tissue slices from each kidney were fixed with $4 \%$ paraformaldehyde (PFA) in PBS overnight $(\mathrm{O} / \mathrm{N})$ at $4^{\circ} \mathrm{C}$ and paraffin embedded for histological assessment and snap-frozen in liquid nitrogen for the molecular analysis. The validation animals ( $n=3-4$ per group) treated with the identical UIR protocol were harvested at the same time points (Day 1, 2, 4, 7, 11 and 14), and the kidney tissue was fixed in $4 \%$ PFA O/N at $4{ }^{\circ} \mathrm{C}$ and snap-frozen in liquid nitrogen for the further analysis. 


\section{scRNAseq Data Analysis}

The generated cDNA libraries were quantified using an hsDNA chip and were sequenced on the Illumina HiSeq 2500 using one flow cell (about 300 million reads) per sample. The raw fastqs were processed by aligning read2 to the mm10 genome using bowtie2-2.2.7 using the $-\mathrm{k} 1$ option. The aligned reads were tagged with their corresponding UMI and barcode from read1. Each aligned read was tagged with its gene name. An expression matrix was generated by counting the number of unique UMls per gene per cell.

Cell-type clusters and markers genes were identified using the R v3.6.1 library Seurat v3.1.0 (Butler et al., 2018; Stuart et al., 2019). All clustering was unsupervised, without driver genes. The influence of the number of unique molecular identifiers was minimized by regression within the ScaleData function. Initial cell filtering selected cells that expressed $>500$ genes. Genes included in the analysis were expressed in a minimum of three cells. Only one read per cell was needed for a gene to be counted as expressed per cell. The resulting gene expression matrix was normalized to 10,000 molecules per cell and log transformed (Macosko et al., 2015). Cells containing high percentages of mitochondrial, $>30 \%$, and hemoglobin genes, $>0.025 \%$ were filtered out. Genes with the highest variability among cells were used for principal components analysis. Cell clusters were determined by the Louvain algorithm. Dimension reduction was performed using the Python implementation of UMAP (Uniform Manifold Approximation and Projection) using significant principal components determined by JackStraw plot. Marker genes were determined for each cluster using the Wilcoxon Rank Sum test within the FindAllMarkers function using genes expressed in a minimum of $10 \%$ of cells and fold change threshold of 1.3. Over/under clustering was verified via gene expression heatmaps. 
Putative signaling interactions between proximal tubules, injured proximal tubules, stromal cells, and mixed-identity cells were assessed. Potential receptor-ligand interactions were found by pairing a celltype expressing a ligand with a cell-type expressing its receptor pair. A receptor or ligand was considered expressed in a cell-type having an average normalized expression of $>0.25$. Receptorligand pairs were determined using the curated receptor-ligand database by the RIKEN FANTOM5 project (Lizio et al., 2015). Receptor-ligand pairings for each cell type were visualized by a chord diagram using the R package circlize (Gu et al., 2014).

Differential gene expression between control proximal tubules, injured proximal tubules and "Mixed Identity Cells" was determined by Wilcoxon Rank Sum test within Seurat's FindMarkers function using a log fold change threshold of 0.5 and an adjusted $p$-value cutoff of 0.01 . Venn diagrams were prepared with the R package VennDiagram (Chen and Boutros, 2011).

\section{RT-qPCR}

Gene expression changes identified by scRNA-seq were validated using the Real-Time Quantitative PCR. Three to six mice from the original scRNA-seq cohort and the additional validation cohort were used per each group. Total RNA was isolated from the homogenized whole kidney lysates with RNA Stat-60 extraction reagent (Amsbio, CS-111) and purified using the GeneJET RNA purification kit (Thermo Fisher Scientific, KO732). The cDNA was synthesized with the iScript Reverse Transcription Supermix (Bio-Rad, 1708841). qPCR was performed with TaqMan universal PCR master mix (Thermo Fisher Scientific, 4304437) on the Applied Biosystems Quant Studio 3 RT PCR system. The TaqMan primers details are presented in the Table S5. The reported Ct values are the mean of two 
replicates of the same cDNA sample. The target gene Ct values were normalized to the eukaryotic 18S rRNA endogenous control and presented as the fold change.

\section{Western Blotting}

We validated the RNA expression changes detected by the scRNAseq with Western blots. Three to six mice from the original ScRNA-seq cohort and the additional validation cohort were used per each group. Total protein was extracted from the homogenized whole kidney lysates using the M-PER mammalian protein extraction reagent (Thermo Fisher Scientific, 78501) supplemented with protease (Thermo Fisher Scientific, 78430) and phosphatase (10 mM Sodium Orthovanadate) inhibitors. $10 \mu \mathrm{g}$ of protein was separated on $4-12 \%$ polyacrylamide gel by SDS electrophoresis, transferred to PVDF membrane, blocked with the Odyssey blocking buffer (LI-COR, 927-40000) and incubated with the target recognizing primary antibodies (Kim1, goat, R\&D Systems, AF1817, $0.25 \mu \mathrm{g} / \mathrm{ml}$; Lcn2, rabbit, $\mathrm{MBL}, \mathrm{JM}-3819-100,0.5 \mu \mathrm{g} / \mathrm{ml}) \mathrm{O} / \mathrm{N}$ at $4{ }^{\circ} \mathrm{C}$. On the next day, the membranes were washed with Tris buffered saline $0.02 \%$ Tween (TBST) and incubated with the secondary fluorescent antibodies for 1 hour at RT in the darkness. The target protein levels were normalized to the endogenous control (GAPDH, mouse, Millipore Sigma, MAB374, 1/500). The membranes were scanned on the LI-COR Odyssey CLx imaging system.

\section{Chromogenic In Situ Hybridization (CISH)}

$\mathrm{CISH}$ riboprobes were generated from the gDNA or CDNA with PCR and labeled with digoxigenin (DIG) using DIG RNA labeling mix (Roche, 11277073910) by in vitro transcription, and the primer sequences are listed in the Table S5. Primer specificity was checked with BLAST analysis. PFA fixed paraffin embedded (PFPE) $6 \mu \mathrm{m}$ kidney sections were subjected to the $\mathrm{CISH}$ protocol as previously 
described (Lan et al., 2001; Xu et al., 2016). On day 1, deparaffinated and dehydrated sections were incubated with Proteinase $\mathrm{K}(1 \mathrm{ug} / \mathrm{ml})$ for $30 \mathrm{~min}$ at $37^{\circ} \mathrm{C}$, fixed in $4 \%$ PFA for $30 \mathrm{~min}$ at room temperature (RT), acetylated in $0.25 \%$ acetic anhydride and hybridized for $14-16$ hours at $70{ }^{\circ} \mathrm{C}$ with the antisense riboprobes. Sense riboprobes were used for the negative controls. All day 1 procedures were performed in the RNase-free conditions. On the second day, sections underwent a series of saline sodium citrate buffer washes and were incubated with the anti-DIG-AP fab fragments (Roche, $11093274910,1: 1000) \mathrm{O} / \mathrm{N}$ at $4{ }^{\circ} \mathrm{C}$, followed by a series of post-hybridization washes and color development with BM Purple (Roche, 11442074001). Color development time varied from several hours to 3-4 days depending on the target gene expression levels. For the immunofluorescent (IF) colabeling, the protocol was modified by adding the primary antibody recognizing the target (Krt8, rabbit, Abcam, EP1628Y, 1/100; Aqp1, mouse, Santa Cruz, sc-25287, 1/50) to the anti-DIG-AP antibody mixture and incubation $\mathrm{O} / \mathrm{N}$ at $4{ }^{\circ} \mathrm{C}$ (Lopez, 2014). Next day, the sections were incubated with the fluorescent secondary antibodies for 1 hour at RT in darkness, followed by the post-hybridization washes and color development, according to the CISH protocol. DAPI (Thermo Fisher Scientific, $62248,1: 1000)$ treatment for nuclei labeling was performed after the color development; the sections were mounted with Vectashield antifade mounting medium (Vector Laboratories, $\mathrm{H}-1000)$. For the double $\mathrm{CISH} / \mathrm{IF}$ staining, treatment with the antisense riboprobe and secondary antibodies only was used as the negative control. For the immunohistochemistry (IHC) staining, the sections were subjected to the endogenous peroxidase quenching, blocking and primary antibody incubation (Cd24a, rat, Abcam, ab64064, 1/100) $\mathrm{O} / \mathrm{N}$ at $4{ }^{\circ} \mathrm{C}$, followed by the signal detection with the ImmPRESS reagent kit with DAB substrate (Chihanga et al., 2018). CISH and IHC images were obtained on the Nikon Ti2 wide-field microscope. RGB images of $\mathrm{CISH} / \mathrm{IHC}$ were taken with an Andor Zyla 4.2 plus camera and a Lumencor LIDA RGB transmitted light source. 
RNAscope probes were purchased from Advanced Cell Diagnostics, Icn (ACD) and are summarized in the Table S5. RNAscope was performed with the Multiplex Fluorescent v2 assay (ACD, 323100) on the freshly sectioned PFPE $6 \mu \mathrm{m}$ kidney sections according to the manufacturer's protocol. Briefly, the deparaffinized and dehydrated kidney sections underwent the endogenous peroxidase quenching, heat-induced target retrieval and protease digestion, followed by the incubation with up to three target riboprobes for 2 hours at $40^{\circ} \mathrm{C}$. All the aforementioned steps were performed in the RNase-free conditions. Next, tyramide signal amplification was performed according to the manufacturer's protocol and conjugated to an Opal dye (PerkinElmer). The sections were treated with DAPI (Thermo Fisher Scientific, 62248, 1:1000) and mounted with Vectashield antifade mounting medium (Vector Laboratories, $\mathrm{H}-1000)$. The negative controls were treated with the signal amplification reagents but no target riboprobes and processed alongside the experimental sections.

\section{Fluorescent Microscopy and Quantitative Image Analysis}

The double $\mathrm{CISH} / \mathrm{IF}$ imaging was performed on a Nikon A1R HD confocal TiE microscope using the galvanometric scanner. The $\mathrm{CISH}$ signal was captured both using a transmitted light detector to detect absorption of photons by the chromogenic substrate and as near-IR fluorescence of the BM purple using a $647 \mathrm{~nm}$ laser for excitation and capturing fluorescence data above $740 \mathrm{~nm}$ with a 740nm long-pass filter (Schumacher et al., 2014; Trinh le et al., 2007). Transmitted images of chromogenic substrate are shown in gray-scale images (Spp1, Figure S6A) and fluorescence of the color development reagent (BM purple) is shown as cyan (Spp1, Figures $5 \mathrm{C}, \mathrm{S} 6 \mathrm{~A}$ ) or magenta (Myh9, Figure 5E).

For the RNAscope single transcript quantification, Z-stacks of $\sim 6 \mu \mathrm{m}$ from multiple (9-12 per sample) focal planes were obtained on 60x water immersion (WI) objective at Nyquist resolution on the Nikon 
Ti-E A1R HD confocal with the resonant scanner. The images were processed with NIS-Elements AR 5.2.00 artificial intelligence denoise algorithm

\section{(https://www.microscope.healthcare.nikon.com/products/confocal-microscopes/a1hd25-a1rhd25/nis-}

elements-ai) and stitched into the single image with NIS-Elements AR stitching tool. All images within an experimental group were obtained with the same optical configuration. We first identified individual renal tubules based on the marker gene expression using the "Manual Surfaces" algorithm in Bitplane Imaris 9.3.1. (Chhipa et al., 2018). Then, we identified the transcripts using the "Spots" algorithm, with identical spot diameter and quality used for the experimental groups and the controls. To quantify the transcript number per tubule, we used the Matlab XT function "split spots into surfaces". We normalized the obtained transcript number to the tubule volume and presented the average of all renal tubules ( $50-70$ tubules) captured in the image.

\section{Statistical Analysis and Reproducibility}

The gene expression changes identified by the scRNAseq were reproduced in the separate cohort of experimental mice of the same strain, age and sex, treated with the identical UIR procedure. Three to six mice per group were used for the qPCR and Western blot analysis. Data were presented as Mean \pm SEM. To determine the statistical significance, $P$ values were generated using one-way ANOVA with Bonferroni and Holm test with * $\mathrm{p}<0.05$ representing the statistically significant difference. The significance is shown compared to the control group. For the RNAscope, 9-12 Z-stack images per animal were analyzed ( $n=1$ animal per time point). Transcript numbers were quantified using Bitplane Imaris 9.3.1. as described above; data were presented as Mean \pm SEM and analyzed with Student's t-test or one-way ANOVA with Bonferroni and Holm test when comparing the two or multiple conditions, respectively; $p<0.05$ represented the statistically significant difference. For the correlation analysis, $\mathrm{R}$ squared was obtained and analyzed with Pearson correlation test; $\mathrm{p}<0.05$ 
represented the statistical significance. The gene ontology (GO) analysis of biological processes was performed in the ToppGene Suite (Chen et al., 2009) with 0.05 p value cutoff. The gene clusters enriched in renal cell populations were generated with the ToppCluster (Kaimal et al., 2010) with Bonferroni correction and $0.05 \mathrm{p}$ value cutoff using the gene level Fruchterman-Reingold algorithm.

\section{Data and Code Availability}

The scRNAseq data were deposited at GEO accession number: GEO: GSE139506.

\section{Supplemental Information}

Figures S1-S6.

Table S1. UIR Day1 vs Control gene expression analysis. Related to Figures 1, 2, 4.

Table S2. Dedifferenriated Prox UIR Day 7 and 11 Marker Gene. Related to Figure 3.

Table S3. "Injured Prox" and "Mixed Identity Cells" Differential Analysis. Related to Figure 3.

Table S4. Receptor-Ligand Interactions in Control and UIR Day 4 Kidney. Related to Figure 6.

Table S5. qPCR primers, riboprobes, $\mathrm{CISH}$ probe primer sequences. Related to Methods.

Movie 1. RNAscope Control proximal tubule, Vim (green), Col18a1 (red), Slc34a1 (purple), 60x Nyquist zoom $0.07 \mu \mathrm{m} / \mathrm{px}$, scale $20 \mu \mathrm{m}$. Related to Figure 6 .

Movie 2. RNAscope UIR Day 1 proximal tubule, Vim (green), Col18a1 (red), Slc34a1 (purple), 60x Nyquist zoom $0.07 \mu \mathrm{m} / \mathrm{px}$, scale $20 \mu \mathrm{m}$. Related to Figure 6. Note elevated Vim and Col18a1 in the Slc34a1 positive tubule.

\section{References}

Adam, M., Potter, A.S., and Potter, S.S. (2017). Psychrophilic proteases dramatically reduce single-cell RNA-seq artifacts: a molecular atlas of kidney development. Development 144, 3625-3632. 
Anders, H.J., Lichtnekert, J., and Allam, R. (2010). Interferon-alpha and -beta in kidney inflammation. Kidney Int 77, 848-854.

Arrondel, C., Vodovar, N., Knebelmann, B., Grunfeld, J.P., Gubler, M.C., Antignac, C., and Heidet, L. (2002). Expression of the nonmuscle myosin heavy chain IIA in the human kidney and screening for MYH9 mutations in Epstein and Fechtner syndromes. J Am Soc Nephrol 13, 65-74.

Basile, D.P., Anderson, M.D., and Sutton, T.A. (2012). Pathophysiology of acute kidney injury. Compr Physiol 2, 1303-1353.

Basson, M.A., Akbulut, S., Watson-Johnson, J., Simon, R., Carroll, T.J., Shakya, R., Gross, I., Martin, G.R., Lufkin, T., McMahon, A.P., et al. (2005). Sprouty1 is a critical regulator of GDNF/RET-mediated kidney induction. Dev Cell 8, 229-239.

Bilir, B., Osunkoya, A.O., Wiles, W.G.t., Sannigrahi, S., Lefebvre, V., Metzger, D., Spyropoulos, D.D., Martin, W.D., and Moreno, C.S. (2016). SOX4 Is Essential for Prostate Tumorigenesis Initiated by PTEN Ablation. Cancer Res 76, 1112-1121.

Bonventre, J.V. (2008). Kidney Injury Molecule-1 (KIM-1): a specific and sensitive biomarker of kidney injury. Scand J Clin Lab Invest Suppl 241, 78-83.

Brunskill, E.W., Park, J.S., Chung, E., Chen, F., Magella, B., and Potter, S.S. (2014). Single cell dissection of early kidney development: multilineage priming. Development 141, 3093-3101.

Butler, A., Hoffman, P., Smibert, P., Papalexi, E., and Satija, R. (2018). Integrating single-cell transcriptomic data across different conditions, technologies, and species. Nat Biotechnol 36, 411-420.

Cacciottolo, M., Belcastro, V., Laval, S., Bushby, K., di Bernardo, D., and Nigro, V. (2011). Reverse engineering gene network identifies new dysferlin-interacting proteins. J Biol Chem 286, 5404-5413.

Challen, G.A., Martinez, G., Davis, M.J., Taylor, D.F., Crowe, M., Teasdale, R.D., Grimmond, S.M., and Little, M.H. (2004). Identifying the molecular phenotype of renal progenitor cells. J Am Soc Nephrol 15, 23442357.

Chen, H., and Boutros, P.C. (2011). VennDiagram: a package for the generation of highly-customizable Venn and Euler diagrams in R. BMC Bioinformatics 12, 35.

Chen, J., Bardes, E.E., Aronow, B.J., and Jegga, A.G. (2009). ToppGene Suite for gene list enrichment analysis and candidate gene prioritization. Nucleic Acids Res 37, W305-311.

Chen, L., Lee, J.W., Chou, C.L., Nair, A.V., Battistone, M.A., Paunescu, T.G., Merkulova, M., Breton, S., Verlander, J.W., Wall, S.M., et al. (2017). Transcriptomes of major renal collecting duct cell types in mouse identified by single-cell RNA-seq. Proc Natl Acad Sci U S A 114, E9989-E9998.

Chhipa, R.R., Fan, Q., Anderson, J., Muraleedharan, R., Huang, Y., Ciraolo, G., Chen, X., Waclaw, R., Chow, L.M., Khuchua, Z., et al. (2018). AMP kinase promotes glioblastoma bioenergetics and tumour growth. Nat Cell Biol 20, 823-835.

Chihanga, T., Ruby, H.N., Ma, Q., Bashir, S., Devarajan, P., and Kennedy, M.A. (2018). NMR-based urine metabolic profiling and immunohistochemistry analysis of nephron changes in a mouse model of hypoxiainduced acute kidney injury. Am J Physiol Renal Physiol 315, F1159-F1173.

Chung, E., Deacon, P., Marable, S., Shin, J., and Park, J.S. (2016). Notch signaling promotes nephrogenesis by downregulating Six2. Development 143, 3907-3913.

Combes, A.N., Phipson, B., Lawlor, K.T., Dorison, A., Patrick, R., Zappia, L., Harvey, R.P., Oshlack, A., and Little, M.H. (2019). Single cell analysis of the developing mouse kidney provides deeper insight into marker gene expression and ligand-receptor crosstalk. Development 146.

Costantini, F., and Kopan, R. (2010). Patterning a complex organ: branching morphogenesis and nephron segmentation in kidney development. Dev Cell 18, 698-712.

Devarajan, P. (2008). Neutrophil gelatinase-associated lipocalin (NGAL): a new marker of kidney disease. Scand J Clin Lab Invest Suppl 241, 89-94.

Devarajan, P. (2010). Neutrophil gelatinase-associated lipocalin: a promising biomarker for human acute kidney injury. Biomark Med 4, 265-280. 
Dieterich, C., Puey, A., Lin, S., Swezey, R., Furimsky, A., Fairchild, D., Mirsalis, J.C., and Ng, H.H. (2009). Gene expression analysis reveals new possible mechanisms of vancomycin-induced nephrotoxicity and identifies gene markers candidates. Toxicol Sci 107, 258-269.

Dieterle, F., Perentes, E., Cordier, A., Roth, D.R., Verdes, P., Grenet, O., Pantano, S., Moulin, P., Wahl, D., Mahl, A., et al. (2010). Urinary clusterin, cystatin C, beta2-microglobulin and total protein as markers to detect drug-induced kidney injury. Nat Biotechnol 28, 463-469.

Djudjaj, S., Papasotiriou, M., Bulow, R.D., Wagnerova, A., Lindenmeyer, M.T., Cohen, C.D., Strnad, P., Goumenos, D.S., Floege, J., and Boor, P. (2016). Keratins are novel markers of renal epithelial cell injury. Kidney Int 89, 792-808.

Fang, J., Wang, H., Liu, Y., Ding, F., Ni, Y., and Shao, S. (2017). High KRT8 expression promotes tumor progression and metastasis of gastric cancer. Cancer Sci 108, 178-186.

Gauer, S., Urbschat, A., Gretz, N., Hoffmann, S.C., Kranzlin, B., Geiger, H., and Obermuller, N. (2016). Kidney Injury Molecule-1 Is Specifically Expressed in Cystically-Transformed Proximal Tubules of the PKD/Mhm (cy/+) Rat Model of Polycystic Kidney Disease. Int J Mol Sci 17.

Grande, M.T., Sanchez-Laorden, B., Lopez-Blau, C., De Frutos, C.A., Boutet, A., Arevalo, M., Rowe, R.G., Weiss, S.J., Lopez-Novoa, J.M., and Nieto, M.A. (2015). Snail1-induced partial epithelial-to-mesenchymal transition drives renal fibrosis in mice and can be targeted to reverse established disease. Nat Med 21,989997.

Gu, Z., Gu, L., Eils, R., Schlesner, M., and Brors, B. (2014). circlize Implements and enhances circular visualization in R. Bioinformatics 30, 2811-2812.

Guo, J., Guan, Q., Liu, X., Wang, H., Gleave, M.E., Nguan, C.Y., and Du, C. (2016). Relationship of clusterin with renal inflammation and fibrosis after the recovery phase of ischemia-reperfusion injury. BMC Nephrol 17, 133.

Hara-Chikuma, M., and Verkman, A.S. (2006). Aquaporin-1 facilitates epithelial cell migration in kidney proximal tubule. J Am Soc Nephrol 17, 39-45.

Harding, S.D., Armit, C., Armstrong, J., Brennan, J., Cheng, Y., Haggarty, B., Houghton, D., Lloyd-MacGilp, S., Pi, X., Roochun, Y., et al. (2011). The GUDMAP database--an online resource for genitourinary research. Development 138, 2845-2853.

Henn, A.D., Berleth, E.S., Mihich, E., and Ehrke, M.J. (2001). Changes in cytosolic and membrane TNF inhibitory protein-B1 (TIP-B1) levels associated with protection from TNF-induced cytotoxicity. FASEB J 15, 1315-1317.

Hochane, M., van den Berg, P.R., Fan, X., Berenger-Currias, N., Adegeest, E., Bialecka, M., Nieveen, M., Menschaart, M., Chuva de Sousa Lopes, S.M., and Semrau, S. (2019). Single-cell transcriptomics reveals gene expression dynamics of human fetal kidney development. PLoS Biol 17, e3000152.

Hoste, E.A.J., Kellum, J.A., Selby, N.M., Zarbock, A., Palevsky, P.M., Bagshaw, S.M., Goldstein, S.L., Cerda, J., and Chawla, L.S. (2018). Global epidemiology and outcomes of acute kidney injury. Nat Rev Nephrol 14, 607-625.

Hsu, R.K., and Hsu, C.Y. (2016). The Role of Acute Kidney Injury in Chronic Kidney Disease. Semin Nephrol 36, 283-292.

Hu, G., and Chen, J. (2013). A genome-wide regulatory network identifies key transcription factors for memory CD8(+) T-cell development. Nat Commun 4, 2830.

Huang, J., Arsenault, M., Kann, M., Lopez-Mendez, C., Saleh, M., Wadowska, D., Taglienti, M., Ho, J., Miao, Y., Sims, D., et al. (2013). The transcription factor Sry-related HMG box-4 (SOX4) is required for normal renal development in vivo. Dev Dyn 242, 790-799.

Humphreys, B.D. (2018). Mechanisms of Renal Fibrosis. Annu Rev Physiol 80, 309-326.

Ivanova, L., Hiatt, M.J., Yoder, M.C., Tarantal, A.F., and Matsell, D.G. (2010). Ontogeny of CD24 in the human kidney. Kidney Int 77, 1123-1131. 
Kaimal, V., Bardes, E.E., Tabar, S.C., Jegga, A.G., and Aronow, B.J. (2010). ToppCluster: a multiple gene list feature analyzer for comparative enrichment clustering and network-based dissection of biological systems. Nucleic Acids Res 38, W96-102.

Kaleta, B. (2019). The role of osteopontin in kidney diseases. Inflamm Res 68, 93-102.

Kang, H.M., Ahn, S.H., Choi, P., Ko, Y.A., Han, S.H., Chinga, F., Park, A.S., Tao, J., Sharma, K., Pullman, J., et al. (2015). Defective fatty acid oxidation in renal tubular epithelial cells has a key role in kidney fibrosis development. Nat Med 21, 37-46.

Khawaja, S., Jafri, L., Siddiqui, I., Hashmi, M., and Ghani, F. (2019). The utility of neutrophil gelatinaseassociated Lipocalin (NGAL) as a marker of acute kidney injury (AKI) in critically ill patients. Biomark Res 7, 4.

Khreba, N.A., Abdelsalam, M., Wahab, A.M., Sanad, M., Elhelaly, R., Adel, M., and El-Kannishy, G. (2019). Kidney Injury Molecule 1 (KIM-1) as an Early Predictor for Acute Kidney Injury in Post-Cardiopulmonary Bypass (CPB) in Open Heart Surgery Patients. Int J Nephrol 2019, 6265307.

Kinnunen, A.I., Sormunen, R., Elamaa, H., Seppinen, L., Miller, R.T., Ninomiya, Y., Janmey, P.A., and Pihlajaniemi, T. (2011). Lack of collagen XVIII long isoforms affects kidney podocytes, whereas the short form is needed in the proximal tubular basement membrane. J Biol Chem 286, 7755-7764.

Kramann, R., Kusaba, T., and Humphreys, B.D. (2015). Who regenerates the kidney tubule? Nephrol Dial Transplant 30, 903-910.

Kudose, S., Hoshi, M., Jain, S., and Gaut, J.P. (2018). Renal Histopathologic Findings Associated With Severity of Clinical Acute Kidney Injury. Am J Surg Pathol 42, 625-635.

Kume, T., Deng, K., and Hogan, B.L. (2000). Murine forkhead/winged helix genes Foxc1 (Mf1) and Foxc2 (Mfh1) are required for the early organogenesis of the kidney and urinary tract. Development 127, 1387-1395.

Kusaba, T., Lalli, M., Kramann, R., Kobayashi, A., and Humphreys, B.D. (2014). Differentiated kidney epithelial cells repair injured proximal tubule. Proc Natl Acad Sci U S A 111, 1527-1532.

Lan, Y., Kingsley, P.D., Cho, E.S., and Jiang, R. (2001). Osr2, a new mouse gene related to Drosophila oddskipped, exhibits dynamic expression patterns during craniofacial, limb, and kidney development. Mech Dev 107, 175-179.

Lazzeri, E., Crescioli, C., Ronconi, E., Mazzinghi, B., Sagrinati, C., Netti, G.S., Angelotti, M.L., Parente, E., Ballerini, L., Cosmi, L., et al. (2007). Regenerative potential of embryonic renal multipotent progenitors in acute renal failure. J Am Soc Nephrol 18, 3128-3138.

Le Clef, N., Verhulst, A., D'Haese, P.C., and Vervaet, B.A. (2016). Unilateral Renal Ischemia-Reperfusion as a Robust Model for Acute to Chronic Kidney Injury in Mice. PLoS One 11, e0152153.

Lee, S., Huen, S., Nishio, H., Nishio, S., Lee, H.K., Choi, B.S., Ruhrberg, C., and Cantley, L.G. (2011). Distinct macrophage phenotypes contribute to kidney injury and repair. J Am Soc Nephrol 22, 317-326.

Linton, J.M., Martin, G.R., and Reichardt, L.F. (2007). The ECM protein nephronectin promotes kidney development via integrin alpha8beta1-mediated stimulation of Gdnf expression. Development 134, 25012509.

Liu, C.Y., Lin, H.H., Tang, M.J., and Wang, Y.K. (2015). Vimentin contributes to epithelial-mesenchymal transition cancer cell mechanics by mediating cytoskeletal organization and focal adhesion maturation. Oncotarget 6, 15966-15983.

Liu, J., Kumar, S., Dolzhenko, E., Alvarado, G.F., Guo, J., Lu, C., Chen, Y., Li, M., Dessing, M.C., Parvez, R.K., et al. (2017). Molecular characterization of the transition from acute to chronic kidney injury following ischemia/reperfusion. JCI Insight 2.

Liu, Y. (2010). New insights into epithelial-mesenchymal transition in kidney fibrosis. J Am Soc Nephrol 21, 212-222.

Lizio, M., Harshbarger, J., Shimoji, H., Severin, J., Kasukawa, T., Sahin, S., Abugessaisa, I., Fukuda, S., Hori, F., Ishikawa-Kato, S., et al. (2015). Gateways to the FANTOM5 promoter level mammalian expression atlas. Genome Biol 16, 22. 
Lopez, M.E. (2014). Combined in situ Hybridization/Immunohistochemistry (ISH/IH) on Free-floating Vibratome Tissue Sections. Bio Protoc 4.

Lovisa, S., Zeisberg, M., and Kalluri, R. (2016). Partial Epithelial-to-Mesenchymal Transition and Other New Mechanisms of Kidney Fibrosis. Trends Endocrinol Metab 27, 681-695.

Macosko, E.Z., Basu, A., Satija, R., Nemesh, J., Shekhar, K., Goldman, M., Tirosh, I., Bialas, A.R., Kamitaki, N., Martersteck, E.M., et al. (2015). Highly Parallel Genome-wide Expression Profiling of Individual Cells Using Nanoliter Droplets. Cell 161, 1202-1214.

Magella, B., Adam, M., Potter, A.S., Venkatasubramanian, M., Chetal, K., Hay, S.B., Salomonis, N., and Potter, S.S. (2018). Cross-platform single cell analysis of kidney development shows stromal cells express Gdnf. Dev Biol 434, 36-47.

McMahon, A.P. (2016). Development of the Mammalian Kidney. Curr Top Dev Biol 117, 31-64.

McMahon, A.P., Aronow, B.J., Davidson, D.R., Davies, J.A., Gaido, K.W., Grimmond, S., Lessard, J.L., Little, M.H., Potter, S.S., Wilder, E.L., et al. (2008). GUDMAP: the genitourinary developmental molecular anatomy project. J Am Soc Nephrol 19, 667-671.

Mishra, J., Dent, C., Tarabishi, R., Mitsnefes, M.M., Ma, Q., Kelly, C., Ruff, S.M., Zahedi, K., Shao, M., Bean, J., et al. (2005). Neutrophil gelatinase-associated lipocalin (NGAL) as a biomarker for acute renal injury after cardiac surgery. Lancet 365, 1231-1238.

Nurnberger, J., Feldkamp, T., Kavapurackal, R., Opazo Saez, A., Becker, J., Horbelt, M., and Kribben, A. (2010). N-cadherin is depleted from proximal tubules in experimental and human acute kidney injury. Histochem Cell Biol 133, 641-649.

Otterpohl, K.L., Hart, R.G., Evans, C., Surendran, K., and Chandrasekar, I. (2017). Nonmuscle myosin 2 proteins encoded by Myh9, Myh10, and Myh14 are uniquely distributed in the tubular segments of murine kidney. Physiol Rep 5.

Pall, T., Pink, A., Kasak, L., Turkina, M., Anderson, W., Valkna, A., and Kogerman, P. (2011). Soluble CD44 interacts with intermediate filament protein vimentin on endothelial cell surface. PLoS One 6, e29305.

Park, J., Shrestha, R., Qiu, C., Kondo, A., Huang, S., Werth, M., Li, M., Barasch, J., and Susztak, K. (2018). Single-cell transcriptomics of the mouse kidney reveals potential cellular targets of kidney disease. Science 360, 758-763.

Paul, M.H., Harvey, R.P., Wegner, M., and Sock, E. (2014). Cardiac outflow tract development relies on the complex function of Sox4 and Sox11 in multiple cell types. Cell Mol Life Sci 71, 2931-2945.

Pavkov, M.E., Harding, J.L., and Burrows, N.R. (2018). Trends in Hospitalizations for Acute Kidney Injury United States, 2000-2014. MMWR Morb Mortal Wkly Rep 67, 289-293.

Prozialeck, W.C., Lamar, P.C., and Appelt, D.M. (2004). Differential expression of E-cadherin, N-cadherin and beta-catenin in proximal and distal segments of the rat nephron. BMC Physiol 4, 10.

Ransick, A., Lindstrom, N.O., Liu, J., Zhu, Q., Guo, J.J., Alvarado, G.F., Kim, A.D., Black, H.G., Kim, J., and McMahon, A.P. (2019). Single-Cell Profiling Reveals Sex, Lineage, and Regional Diversity in the Mouse Kidney. Dev Cell 51, 399-413 e397.

Rinn, J.L., Rozowsky, J.S., Laurenzi, I.J., Petersen, P.H., Zou, K., Zhong, W., Gerstein, M., and Snyder, M. (2004). Major molecular differences between mammalian sexes are involved in drug metabolism and renal function. Dev Cell 6, 791-800.

Ruan, H., Yang, H., Wei, H., Xiao, W., Lou, N., Qiu, B., Xu, G., Song, Z., Xiao, H., Liu, L., et al. (2017). Overexpression of SOX4 promotes cell migration and invasion of renal cell carcinoma by inducing epithelialmesenchymal transition. Int J Oncol 51, 336-346.

Schmidt-Ott, K.M., Mori, K., Li, J.Y., Kalandadze, A., Cohen, D.J., Devarajan, P., and Barasch, J. (2007). Dual action of neutrophil gelatinase-associated lipocalin. J Am Soc Nephrol 18, 407-413.

Schumacher, J.A., Zhao, E.J., Kofron, M.J., and Sumanas, S. (2014). Two-color fluorescent in situ hybridization using chromogenic substrates in zebrafish. Biotechniques 57, 254-256. 
She, Z.Y., and Yang, W.X. (2015). SOX family transcription factors involved in diverse cellular events during development. Eur J Cell Biol 94, 547-563.

Shieh, J.M., Tsai, Y.J., Chi, J.C., and Wu, W.B. (2019). TGFbeta mediates collagen production in human CRSsNP nasal mucosa-derived fibroblasts through Smad2/3-dependent pathway and CTGF induction and secretion. J Cell Physiol 234, 10489-10499.

Simon, N., and Hertig, A. (2015). Alteration of Fatty Acid Oxidation in Tubular Epithelial Cells: From Acute Kidney Injury to Renal Fibrogenesis. Front Med (Lausanne) 2, 52.

Singer, E., Schrezenmeier, E.V., Elger, A., Seelow, E.R., Krannich, A., Luft, F.C., and Schmidt-Ott, K.M. (2016). Urinary NGAL-Positive Acute Kidney Injury and Poor Long-term Outcomes in Hospitalized Patients. Kidney Int Rep 1, 114-124.

Sirin, Y., and Susztak, K. (2012). Notch in the kidney: development and disease. J Pathol 226, 394-403.

Skrypnyk, N.I., Harris, R.C., and de Caestecker, M.P. (2013). Ischemia-reperfusion model of acute kidney injury and post injury fibrosis in mice. J Vis Exp.

Sohn, M., Shin, S., Yoo, J.Y., Goh, Y., Lee, I.H., and Bae, Y.S. (2018). Ahnak promotes tumor metastasis through transforming growth factor-beta-mediated epithelial-mesenchymal transition. Sci Rep 8, 14379.

Stuart, T., Butler, A., Hoffman, P., Hafemeister, C., Papalexi, E., Mauck, W.M., 3rd, Hao, Y., Stoeckius, M., Smibert, P., and Satija, R. (2019). Comprehensive Integration of Single-Cell Data. Cell 177, 1888-1902 e1821.

Sun, W.Y., Bai, B., Luo, C., Yang, K., Li, D., Wu, D., Feletou, M., Villeneuve, N., Zhou, Y., Yang, J., et al. (2018). Lipocalin-2 derived from adipose tissue mediates aldosterone-induced renal injury. JCI Insight 3.

Takaori, K., Nakamura, J., Yamamoto, S., Nakata, H., Sato, Y., Takase, M., Nameta, M., Yamamoto, T., Economides, A.N., Kohno, K., et al. (2016). Severity and Frequency of Proximal Tubule Injury Determines Renal Prognosis. J Am Soc Nephrol 27, 2393-2406.

Tan, H.S., Jiang, W.H., He, Y., Wang, D.S., Wu, Z.J., Wu, D.S., Gao, L., Bao, Y., Shi, J.Z., Liu, B., et al. (2017). KRT8 upregulation promotes tumor metastasis and is predictive of a poor prognosis in clear cell renal cell carcinoma. Oncotarget 8, 76189-76203.

Tavazoie, S.F., Alarcon, C., Oskarsson, T., Padua, D., Wang, Q., Bos, P.D., Gerald, W.L., and Massague, J. (2008). Endogenous human microRNAs that suppress breast cancer metastasis. Nature 451, 147-152.

Tokonami, N., Takata, T., Beyeler, J., Ehrbar, I., Yoshifuji, A., Christensen, E.I., Loffing, J., Devuyst, O., and Olinger, E.G. (2018). Uromodulin is expressed in the distal convoluted tubule, where it is critical for regulation of the sodium chloride cotransporter NCC. Kidney Int 94, 701-715.

Trinh le, A., McCutchen, M.D., Bonner-Fraser, M., Fraser, S.E., Bumm, L.A., and McCauley, D.W. (2007). Fluorescent in situ hybridization employing the conventional NBT/BCIP chromogenic stain. Biotechniques 42, 756-759.

Wang, P., Chen, Y., Yong, J., Cui, Y., Wang, R., Wen, L., Qiao, J., and Tang, F. (2018). Dissecting the Global Dynamic Molecular Profiles of Human Fetal Kidney Development by Single-Cell RNA Sequencing. Cell Rep 24, 3554-3567 e3553.

Wu, H., Lai, C.F., Chang-Panesso, M., and Humphreys, B.D. (2019). Proximal Tubule Translational Profiling during Kidney Fibrosis Reveals Proinflammatory and Long Noncoding RNA Expression Patterns with Sexual Dimorphism. J Am Soc Nephrol.

Wu, W., Zaal, E.A., Berkers, C.R., Lemeer, S., and Heck, A.J.R. (2018). CTGF/VEGFA-activated Fibroblasts Promote Tumor Migration Through Micro-environmental Modulation. Mol Cell Proteomics 17, 1502-1514.

Xu, J., Liu, H., Lan, Y., Aronow, B.J., Kalinichenko, V.V., and Jiang, R. (2016). A Shh-Foxf-Fgf18-Shh Molecular Circuit Regulating Palate Development. PLoS Genet 12, e1005769.

Yang, L., Besschetnova, T.Y., Brooks, C.R., Shah, J.V., and Bonventre, J.V. (2010). Epithelial cell cycle arrest in G2/M mediates kidney fibrosis after injury. Nat Med 16, 535-543, 531p following 143.

Yu, J., Valerius, M.T., Duah, M., Staser, K., Hansard, J.K., Guo, J.J., McMahon, J., Vaughan, J., Faria, D., Georgas, K., et al. (2012). Identification of molecular compartments and genetic circuitry in the developing mammalian kidney. Development 139, 1863-1873. 
Yu, L., Moriguchi, T., Souma, T., Takai, J., Satoh, H., Morito, N., Engel, J.D., and Yamamoto, M. (2014). GATA2 regulates body water homeostasis through maintaining aquaporin 2 expression in renal collecting ducts. Mol Cell Biol 34, 1929-1941.

Zhang, J., Han, J., Liu, J., Liang, B., Wang, X., and Wang, C. (2017a). Clinical significance of novel biomarker NGAL in early diagnosis of acute renal injury. Exp Ther Med 14, 5017-5021.

Zhang, L.Y., Ge, X.L., Li, Z., Tang, Y.J., Xiong, Y.Y., Li, X.J., Liu, J.F., Wanggou, S.Y., Li, C.T., Yang, K., et al. (2017b). Fibroblasts play a potential role in bone destruction via osteopontin related caldesmon expression and polymerization in human non-functioning pituitary adenomas. Sci Rep 7, 17523. 